\title{
Two manuscripts, one by Routley, one by Meyer: The origins of the Routley-Meyer semantics for relevance logics
}

\author{
Katalin Bimbó ${ }^{1}$, J. Michael Dunn ${ }^{2}$, and Nicholas Ferenz ${ }^{1}$ \\ ${ }^{2}$ School of Informatics, Computing and Engineering and \\ Department of Philosophy, Indiana University \\ and ${ }^{1}$ Department of Philosophy, University of Alberta \\ bimbo@ualberta.ca,dunn@indiana.edu,ferenz@ualberta.ca
}

\begin{abstract}
A ternary relation is often used nowadays to interpret an implication connective of a logic, a practice that became dominant in the semantics of relevance logics. This paper examines two early manuscripts - one by Routley, another by Meyer - in which they were developing set-theoretic semantics for various relevance logics. A standard presentation of a ternary relational semantics for, let us say, the logic of relevant implication $\mathbf{R}$ is quite illuminating, yet the invention of this semantics was fraught with false starts. Meyer's manuscript, in which he builds on some ideas from Routley's manuscript, essentially contains a relational semantics for which $\mathbf{R}^{\circ \boldsymbol{t}}$ is sound and complete.
\end{abstract}

Keywords: entailment, operational semantics, relational semantics, relevant implication, relevance logic

\section{Introduction}

It is now a routine part of the study of classical logic to recognize that there are both proofs and models (interpretations), and that these are connected by soundness and completeness. Soundness says that every provable formula (theorem) is valid, i.e., true in every model, and completeness states the converse. But it was not always routine. When Frege first presented classical logic in his Begriffsschrift in 1879, he did it just by proofs, i.e., he gave axioms together with rules for proving theorems from these. The interpretations were lying there in the background, and for just the propositional 
logic (no quantifiers) they certainly could be seen to be made using Frege's two truth values, the True and the False. But it was not until Emil Post (in 1921) proved completeness for propositional logic that proofs and validity came together. ${ }^{1}$

Modal logic was also just a set formulas with axioms, and rules for many years before having a semantics. There was always the vague link between the necessity/possibility operators and "possible worlds." But Kripke [17] "mathematized" these so as to give an account in terms of "frames" $\langle K, R\rangle$, where $K$ is a set of "worlds" and $R$ is a binary relation of "relative possibility" on $K$. We put quotes around the key words, because in fact $K$ could be any set at all, and $R$ could then be any relation on $K$, subject to various formal requirements such as reflexivity, symmetry, transitivity, just to name a few. Depending on the requirements, various modal logics could be obtained. It is interesting that this "possible-world semantics" for modal logic (as it is often called) is also frequently called the "Kripke semantics." In fact, a number of logicians independently developed similar ideas, as documented by Copeland [5] and Goldblatt [16].

The so-called "Routley-Meyer semantics" for relevance logic has a similar story, which has been told several times, often by one or more of us see Dunn [8], Anderson, Belnap and Dunn [1], Bimbó and Dunn [4]. Here we will be focusing on two draft papers (never published). The first of these was hand-written by Richard Routley, and titled "Semantical Analysis of Entailment and Relevant Implication: I," and dates from late 1970 or early $1971 .^{2}$ The second of these was typed by Robert K. Meyer in response to Routley's paper and dates from early 1971. It was titled "Semantical Completeness of Relevant Implication." 3 These papers have informally been referred to as "Routley's Dead Sea Scrolls," and "From the Desk of Robert K. Meyer" (that is what Meyer had typed in the upper right-hand corner).

\footnotetext{
${ }^{1}$ Though Zach [29] argues that completeness and some other results were first obtained by D. Hilbert and P. Bernays in 1918.

${ }^{2}$ It is fair to say that Routley's penmanship could have been improved, but it has been carefully transcribed by one of us (NF). See Routley [24] and Routley [25].

Routley's name was, well, "Routley" until he changed it to "Sylvan" in 1983. In this paper, we shall refer to him as Routley, because the term "Routley-Meyer semantics" has been chiseled in stone, so to speak.

${ }^{3}$ We are grateful to Alasdair Urquhart for giving us a scan of his copy of this document which was sent to him by Routley. We also thank Michael McRobbie for a draft of a paper written by Meyer in response to Routley's draft paper, which Routley had sent to Meyer. We also thank McRobbie for a copy of an incomplete draft of a paper by Meyer mentioned in the next paragraph, which Meyer had presumably shared with Routley. McRobbie had kept these from when he served as a research assistant to Routley.
}

Australasian Journal of Logic (15:2) 2018, Article no. 3.2 
Meyer drafted at least the beginnings of another paper - probably, in 1972 while on the faculty at the University of Toronto - focused on developing an operational semantics for the negation-free fragment of $\mathbf{R}$ (i.e., $\mathbf{R}_{+}$). The type-written copy we saw ends on p. 15 (in the middle of a sentence), and p. 14 is missing. Although there are numerous citations and numberings for endnotes, the bibliography and the endnotes themselves are missing too. We do not know whether Meyer actually finished the paper and we happened to just have part of it, but it seems likely to us that he just set it aside at some point. There are many manuscripts by Meyer that he never completed (and an amazing number of others that he did complete, and publish).

We mention this paper so as to quote Meyer's own impression of the independent contributions made by others towards the creation of the ternary relational semantics for $\mathbf{R} .^{4}$

In the semantical analysis of $\mathrm{R}$, two approaches have predominated. Urquhart in [4] takes off from the Anderson-Belnap natural deduction formulation of $\mathrm{R}$ in [5] and proposes a semilattice semantics for the implicational fragment $\mathrm{R}_{\mathrm{I}}$ of $\mathrm{R}$ (and for related implicational logics). ${ }^{2}$ Routley and the author, on the other hand, took essentially the algebraic analysis of $\mathrm{R}$ initiated by Dunn in [6] as a starting point to develop a Kripke-style relational semantics for $\mathrm{R}$ and related logics, in [7] and succeeding papers. ${ }^{3}$ Fine's work in [8], though quite independent, contains some of the features peculiar to each approach, though it is closer both in spirit and technique to [7] than to [4]. In turn, the present essay will more nearly resemble [8]; while neither the author nor Fine has had much causal influence on the other, there is an evident kinship in style of logical imagination. (I take the occasion to express awe at the speed and precision with which Fine gets to the heart of things; what he saw was that, in relevance logics, it is the structuring of theories which is everything, a point that Routley and I also drummed away on in [7], and which also plays a crucial role below.)

What Meyer has been too generous to say is that the so-called "RoutleyMeyer semantics" is misnamed. Not because someone else anticipated the general idea, but because Routley got it wrong, at least originally. ${ }^{5}$ Meyer

\footnotetext{
${ }^{4}$ The numbers in the quotation are as in the original typescript.

${ }^{5}$ Both of these might be true, see Dunn and Bimbó [4].
} 
is the first one to actually implement the ternary semantics and get completeness. It is of course more complicated than that (everything is), and Routley did have the idea of the ternary semantics. But the Routley-Meyer publications are the result of two drafts, one by Routley and the other by Meyer. In the first the idea of ternary semantics is briefly mentioned, but on a single page along with a binary operational semantics. It is the latter that is pursued, and in the end does not work. ${ }^{6}$ Meyer is the one who took up the idea of the ternary relational semantics and succeeded. Perhaps, it should be called the "Meyer-Routley semantics for relevance logic"? We do not want to push this, because the source of Meyer's pursuit was that half-page of Routley's manuscript. In fact, the message we want to give, and leave, is the fruitfulness of collaboration.

One of us (JMD) was a colleague of Meyer in the Department of Philosophy at Indiana University, when Meyer received from Routley a copy of his manuscript, early in 1971 (probably, January). Meyer and Dunn discussed the manuscript, which focused on the operational way of expressing the semantics as we shall later discuss. Routley's manuscript was ambitious. Meyer told JMD at the time that Richard "claimed the waterfront." 7 Besides presenting various versions of semantics for the propositional relevance $\operatorname{logics} \mathbf{R}$ and $\mathbf{E}$ and their variants, there was also a proposed semantics for first-order variants, a proposed Gentzen system for $\mathbf{R}_{+}$, a section on decidability, tableaux systems - the list goes on and on. The knowledgable reader will realize that the first Gentzen system for $\mathbf{R}_{+}$was created by Mints [22] and Dunn [7] (not knowing of each other's work) and that a whole range of relevance logics (including $\mathbf{R}$ and $\mathbf{E}$ ) were shown undecidable by Urquhart [28]. Also in the listed "Contents" is a section on "Quantification," but it is not in the actual manuscript. ${ }^{8}$ Fine [15] showed that a constant domain semantics (proposed by Routley and Meyer, Urquhart, and Fine himself) was in fact incomplete.

\footnotetext{
${ }^{6}$ Fine [14] and Maksimova [18] independently (from each other, and from Routley and Meyer) produced a variation of the Routley-Meyer semantics, which combines the operational and relational approaches. KB and JMD discuss this in [4].

${ }^{7}$ This is a variant of the well-known phrase "covers the waterfront." Meyer later used this in his obituary of Routley, when he said "In philosophy Richard "covered the waterfront," tackling topics in (among others) logic, epistemology, metaphysics, ethics and environmental philosophy." (Bulletin of Symbolic Logic, 4 (1998), p. 338)

${ }^{8}$ It is listed as on p. 95-96/68, but that is a "Covering Note" written by Routley and is not an actual part of the manuscript itself.

The references to Routley's manuscript include two page numbers separated by a slash. The first number refers to the original handwritten manuscript [24], the second one refers to the more easily accessible transcript [25].
}

Australasian Journal of Logic (15:2) 2018, Article no. 3.2 
But getting back to the operational semantics, which is Routley's point of focus, Meyer and Dunn became aware of Urquhart's so-called semi-lattice semantics, developed about the same time. They came to realize it was essentially the same as Routley's operational semantics (see [4] for more details, both historical and technical). Meyer and Dunn saw a comparison to Urquhart's semi-lattice semantics, which they had only recently become aware of and already had shown to be incomplete. The problem was that the following formula is valid in the operational semantics, but not provable in $\mathbf{R}^{9}$

$$
((\mathcal{A} \rightarrow(\mathcal{B} \vee \mathcal{C})) \wedge(\mathcal{B} \rightarrow \mathcal{D})) \rightarrow(\mathcal{A} \rightarrow(\mathcal{D} \vee \mathcal{C}))
$$

It turns out (see below) that there is also a problem for soundness, depending on how one treats the semantics of negation among the various choices Routley presents. But that, we think, was not known to Meyer who seized the opportunity to correct matters by using the ternary relation, which was mentioned by Routley, even though he clearly favored the binary operation. A binary operation is clearly a special case of a ternary relation, but not the other way around.

It is worth remarking that Routley had as the very last leaf (p. 9596/68) of his manuscript what he labeled a "Covering Note" written by him for those to whom he sent the manuscript. ${ }^{10}$ It starts: "There are several gaps in the argument, and no doubt many invalid moves - I should be grateful if you would point out all those you think I have failed to see." He then goes on to list eight "chief gaps \& deficiencies." We will talk about some of these later, but for now we just want to comment on the courage and openness that Routley showed in sharing his very original, but incomplete thoughts. In particular, it left the door open for Meyer to come in and fix some things.

\section{Entailment and Relevant Implication}

Routley had a very ambitious task when preparing his manuscript, namely, he wanted to provide semantics for several logics in one fell swoop. His manuscript is long and rich, and we will not try to work through all the

\footnotetext{
${ }^{9}$ Note though that it would be a theorem of $\mathbf{R}$ if one were to add $\mathcal{C} \rightarrow \mathcal{C}$ as an additional conjunct in the antecedent. Incidentally, the counterexample can be simplified to $((\mathcal{A} \rightarrow(\mathcal{B} \vee \mathcal{C})) \wedge(\mathcal{B} \rightarrow \mathcal{C})) \rightarrow(\mathcal{A} \rightarrow \mathcal{C})$. Cf. [8, p. 196]

${ }^{10}$ We do not know exactly who was sent a copy other than Meyer and Urquhart. Dunn was not, though he was able to read Meyer's copy.
}

Australasian Journal of Logic (15:2) 2018, Article no. 3.2 
ideas in it. Rather, we will focus on some semantics he proposed in the manuscript and compare them to work done by Urquhart and Meyer for the same logics - at around the same time.

To start with, we define the logics, which will be considered here, as axiomatic systems. An axiom system, as a rule, is not unique for any given logic. We aim to provide a concise formulation for $\mathbf{R}$, from which some other logics, $\mathbf{R}_{+}, \mathbf{E}$ and $\mathbf{E}_{+}$result by omitting some axiom schemas. We will use the same notation for all the logics, and we will not follow the notations used by Routley or Meyer. We use $\rightarrow$ for implication, $\wedge$ for conjunction, $\vee$ for disjunction and $\sim$ for negation, and we denote the (denumerably many) propositional variables by $p, q, r, p_{0}, p_{1}, \ldots$ and the formulas by $\mathcal{A}, \mathcal{B}, \mathcal{C}, \ldots{ }^{11}$

$$
\begin{aligned}
& \mathrm{A} 1 \mathcal{A} \rightarrow \mathcal{A} \\
& \mathrm{A} 2(\mathcal{A} \rightarrow \mathcal{B}) \rightarrow((\mathcal{C} \rightarrow \mathcal{A}) \rightarrow(\mathcal{C} \rightarrow \mathcal{B})) \\
& \mathrm{A} 3(\mathcal{A} \rightarrow(\mathcal{A} \rightarrow \mathcal{B})) \rightarrow(\mathcal{A} \rightarrow \mathcal{B}) \\
& \mathrm{A} 4(\mathcal{A} \rightarrow(\mathcal{B} \rightarrow \mathcal{C})) \rightarrow(\mathcal{B} \rightarrow(\mathcal{A} \rightarrow \mathcal{C})) \\
& \mathrm{A} 5(\mathcal{A} \rightarrow((\mathcal{B} \rightarrow \mathcal{D}) \rightarrow \mathcal{C})) \rightarrow((\mathcal{B} \rightarrow \mathcal{D}) \rightarrow(\mathcal{A} \rightarrow \mathcal{C})) \\
& \mathrm{A} 6(\mathcal{A} \wedge \mathcal{B}) \rightarrow \mathcal{A} \\
& \mathrm{A} 7(\mathcal{A} \wedge \mathcal{B}) \rightarrow \mathcal{B} \\
& \mathrm{A} 8((\mathcal{C} \rightarrow \mathcal{A}) \wedge(\mathcal{C} \rightarrow \mathcal{B})) \rightarrow(\mathcal{C} \rightarrow(\mathcal{A} \wedge \mathcal{B})) \\
& \mathrm{A} 9(((\mathcal{A} \rightarrow \mathcal{A}) \wedge(\mathcal{B} \rightarrow \mathcal{B})) \rightarrow \mathcal{C}) \rightarrow \mathcal{C} \\
& \mathrm{A} 10 \mathcal{A} \rightarrow(\mathcal{A} \vee \mathcal{B}) \\
& \mathrm{A} 11 \mathcal{A} \rightarrow(\mathcal{B} \vee \mathcal{A}) \\
& \mathrm{A} 12((\mathcal{A} \rightarrow \mathcal{C}) \wedge(\mathcal{B} \rightarrow \mathcal{C})) \rightarrow((\mathcal{A} \vee \mathcal{B}) \rightarrow \mathcal{C}) \\
& \mathrm{A} 13(\mathcal{A} \wedge(\mathcal{B} \vee \mathcal{C})) \rightarrow((\mathcal{A} \wedge \mathcal{B}) \vee(\mathcal{A} \wedge \mathcal{C})) \\
& \mathrm{A} 14(\mathcal{A} \rightarrow \sim \mathcal{A}) \rightarrow \sim \mathcal{A} \\
& \mathrm{A} 15(\mathcal{A} \rightarrow \sim \mathcal{B}) \rightarrow(\mathcal{B} \rightarrow \sim \mathcal{A}) \\
& \mathrm{A} 16 \sim \mathcal{A} \rightarrow \mathcal{A}
\end{aligned}
$$

\footnotetext{
${ }^{11}$ We assume familiarity with basic concepts such as well-formed formulas and proofs in an axiomatic calculus, etc. See e.g., [8] or [2], and references therein.
} 
$\mathrm{R} 1 \mathcal{A}$ and $\mathcal{A} \rightarrow \mathcal{B}$ imply $\mathcal{B}$

R2 $\mathcal{A}$ and $\mathcal{B}$ imply $\mathcal{A} \wedge \mathcal{B}$

Sometimes it is convenient (or outright useful) to have $\boldsymbol{t}$ (truth) and $\circ$ (fusion) in the logic too. To all the logics that we consider, these logical particles may be added conservatively by some of the following axioms and rules. When these particles are in the language, we will stress this by adding $\boldsymbol{t}$ or $\circ$ (as appropriate) in the superscript in the label of the logic.

$\mathrm{A} 17(\boldsymbol{t} \rightarrow \boldsymbol{t}) \rightarrow \boldsymbol{t}$

A18 $t$

R3 If $\mathcal{A}$ is a theorem, then so is $t \rightarrow \mathcal{A}$.

R4 If $\boldsymbol{t} \rightarrow \mathcal{A}$ is a theorem, then so is $\mathcal{A}$.

R5 If $\mathcal{A} \rightarrow(\mathcal{B} \rightarrow \mathcal{C})$ is a theorem, then so is $(\mathcal{A} \circ \mathcal{B}) \rightarrow \mathcal{C}$.

R6 If $(\mathcal{A} \circ \mathcal{B}) \rightarrow \mathcal{C}$ is a theorem, then so is $\mathcal{A} \rightarrow(\mathcal{B} \rightarrow \mathcal{C})$.

Definition 1.1. The logic $\mathbf{R}$ comprises (A1)-(A16) and rules (R1)-(R2). $\mathbf{E}$ is obtained by omitting (A4). The positive fragments of these logics, $\mathbf{R}_{+}$ and $\mathbf{E}_{+}$, respectively, omit (A14)-(A16). $\mathbf{R}_{\wedge}$ and $\mathbf{E}_{\wedge}$ exclude (A10)-(A13) too. $\mathbf{R}^{t}$ and $\mathbf{E}^{t}$ add axioms (A17)-(A18) and rules (R3)-(R4) to $\mathbf{R}$ and $\mathbf{E}$, repectively. The implicational fragments $\mathbf{R}_{\rightarrow}$ and $\mathbf{E}_{\rightarrow}$ are axiomatized by (A1)-(A5), (R1), and by (A1)-(A3), (A5) and (R1). A logic with fusion $\mathbf{X}^{\circ}$ is the logic $\mathbf{X}$ with (R5)-(R6) added, where $\mathbf{X}$ is any of the previously mentioned logics.

\section{Routley's "Dead Sea Scrolls"}

The so-called semi-lattice or operational semantics was published in Urquhart [27]. To anchor our subsequent discussion, we briefly recall this.

\subsection{Urquhart's Semantics for Some Fragments of E and R}

Urquhart's semi-lattice semantics for the implicational fragment $\mathbf{R}_{\rightarrow}$ and the positive fragment $\mathbf{R}_{+}$of $\mathbf{R}$ is based on the idea of combining pieces of information. One can combine any two pieces of information $X$ and $Y$ to form the piece of information $X \cup Y$, which contains the information in both 
$X$ and $Y$. Moreover, according to his concept of "piece of information," the operation of combining information is idempotent, associative, and commutative. The empty piece of information, denoted by 0 , is included and satisfies $X \cup 0=X$. That is, the set of pieces of information $S$ containing 0 and closed under $\cup$ is a semi-lattice.

Furthermore, from each piece of information $X$ there is a (possibly null) set of atomic propositions that may be concluded. These are the atomic propositions determined by $X$. A valuation is defined, which assigns to each atomic proposition $p$ and each piece of information $X$ either $T$ (if $X$ determines $p$ ) or $F$ (if $X$ does not determine $p$ ). That is, $v(p, X)=T$, if $X$ determined $p$, and $v(p, X)=F$, otherwise. For convenience, we will write $X, v \vDash p$ in place of $v(p, X)=T$ and $X, v \not \models p$ in place of $v(p, X)=F$. For simplicity, we shall drop the $v$ whenever doing so does not create ambiguity.

Definition 2.1. A model for $\mathbf{R}_{\rightarrow}$ or $\mathbf{R}_{+}$is a semi-lattice with 0 together with a valuation extended to the appropriate connectives. The valuation is defined for the formulas of $\mathbf{R}_{\rightarrow}$ by conditions (CR1)-(CR2), and for $\mathbf{R}_{+}$by conditions (CR1)-(CR4).

CR1 $X \vDash p \quad$ iff $\quad p$ is determined by $X$;

CR2 $X \vDash \mathcal{A} \rightarrow \mathcal{B} \quad$ iff $\quad$ for all $Y$, either $Y \not \models \mathcal{A}$ or $X \cup Y \vDash \mathcal{B}$;

CR3 $X \vDash \mathcal{A} \wedge \mathcal{B} \quad$ iff $\quad X \vDash \mathcal{A}$ and $X \vDash \mathcal{B}$;

$\mathrm{CR} 4 \quad X \vDash \mathcal{A} \vee \mathcal{B} \quad$ iff $\quad X \vDash \mathcal{A}$ or $X \vDash \mathcal{B}$.

Let $\mathfrak{L}$ be $\mathbf{R}_{\rightarrow}$ or $\mathbf{R}_{+}$; a formula $\mathcal{A}$ is $\mathfrak{L}$-valid when $0 \vDash \mathcal{A}$ for every model for $\mathfrak{L}$. As a result, a formula is valid if and only if it is a theorem of $\mathbf{R}_{\rightarrow}$ (see [27, pp. 161-2]). However, while this semantics is sound for $\mathbf{R}_{+}$, it is incomplete. The formula (already mentioned above) $((\mathcal{A} \rightarrow(\mathcal{B} \vee \mathcal{C})) \wedge(\mathcal{B} \rightarrow$ $\mathcal{D})) \rightarrow(\mathcal{A} \rightarrow(\mathcal{D} \vee \mathcal{C}))$ is valid, but is not a theorem of $\mathbf{R}_{+} \cdot{ }^{12}$

For the implicational and positive fragments of $\mathbf{E},\left(\mathbf{E}_{\rightarrow}\right.$ and $\mathbf{E}_{+}$, respectively), a set of possible worlds is introduced. The set of worlds $W$, the members of which will be denoted by $w$ with or without a subscript, is ordered by a reflexive and transitive relation, denoted by $\leq$. The valuation for atomic propositions is altered to $v\left(p, X, w_{i}\right)=T$ if $p$ is determined by the piece of information $X$, given the facts in world $w_{i}$, and $v\left(p, X, w_{i}\right)=F$, otherwise. Again, we will typically drop $v$, and use the convenient notation $X, w_{i} \vDash \mathcal{A}$.

\footnotetext{
${ }^{12}$ Urquhart credits this counterexample to Dunn and Meyer — see [27, p. 163].
} 
Definition 2.2. A model for $\mathfrak{L}$ (where $\mathfrak{L}$ is $\mathbf{E}_{\rightarrow}$ or $\mathbf{E}_{+}$) is a semi-lattice with 0 , a set of possible worlds ordered by $\leq$, and a valuation extended to the appropriate connectives. The valuation is defined for the formulas of $\mathbf{E}_{\rightarrow}$ by conditions (CE1)-(CE2), and for $\mathbf{E}_{+}$by conditions (CE1)-(CE4).

CE1 $X, w_{i} \vDash p$ iff $p$ is determined by $X$, given the facts obtaining at $w_{i}$

CE2 $X, w_{i} \vDash \mathcal{A} \rightarrow \mathcal{B}$ iff for all $Y$ and for all $w_{j}$ such that $w_{i} \leq w_{j}$, either $Y, w_{j} \not \models \mathcal{A}$ or $X \cup Y, w_{j} \vDash \mathcal{B}$;

CE3 $X, w_{i} \vDash \mathcal{A} \wedge \mathcal{B} \quad$ iff $\quad X, w_{i} \vDash \mathcal{A}$ and $X, w_{i} \vDash \mathcal{B}$;

$\mathrm{CE} 4 \quad X, w_{i} \vDash \mathcal{A} \vee \mathcal{B} \quad$ iff $\quad X, w_{i} \vDash \mathcal{A}$ or $X, w_{i} \vDash \mathcal{B}$.

A formula $\mathcal{A}$ is $\mathfrak{L}$-valid if and only if, for every model for $\mathfrak{L}, 0, w_{i} \vDash \mathcal{A}$, for every $w_{i} \in W$. As in the case of the fragments of $\mathbf{R}$, the defined semantics is sound and complete for $\mathbf{E}_{\rightarrow}$. However, the semantics is sound but incomplete for the positive fragment $\mathbf{E}_{+}$. Again, the formula we mentioned above serves as a counterexample. Another formula that is also a counterexample is $((\mathcal{A} \rightarrow \mathcal{A}) \wedge((\mathcal{A} \wedge \mathcal{B}) \rightarrow \mathcal{C}) \rightarrow(\mathcal{A} \rightarrow(\mathcal{B} \vee \mathcal{C}))) \rightarrow(\mathcal{A} \rightarrow \mathcal{C})$, which is mentioned in $[4$, p. 573$]$.

Urquhart discusses several attempts to deal with negation, each of which fails. The first is to simply require that $X \vDash \sim \mathcal{A}$ iff $X \not \models \mathcal{A}$. However, this validates all instances of $(\mathcal{B} \wedge \sim \mathcal{B}) \rightarrow \mathcal{A}$ and $\mathcal{A} \rightarrow(\mathcal{B} \vee \sim \mathcal{B})$. The second attempt is to add a function $C$ to the semi-lattice and require that the semi-lattice is closed under $C$, and furthermore, require that $C C X=X$ and $C 0=0$ hold. The valuation for $\mathbf{R}$ or $\mathbf{E}$ is thus defined by adding a condition (CRC) or (CEC) to the semantics for $\mathbf{R}_{+}$or $\mathbf{E}_{+}$, respectively.

$$
\begin{aligned}
& \mathrm{CRC} \quad X \vDash \sim \mathcal{A} \quad \text { iff } \quad C X \not \models \mathcal{A} ; \\
& \mathrm{CEC} \quad X, w_{i} \vDash \sim \mathcal{A} \quad \text { iff } \quad C X, w_{i} \not \models \mathcal{A} .
\end{aligned}
$$

As a result, implication-free, two-valued tautologies, the laws of double negation, and the De Morgan's Laws are all valid. However, the formulas $(\mathcal{A} \rightarrow \sim \mathcal{A}) \rightarrow \sim \mathcal{A}$ and $(\mathcal{A} \rightarrow \mathcal{B}) \rightarrow(\sim \mathcal{B} \rightarrow \sim \mathcal{A})$ are both invalid (see $[27$, pp. 164-5]).

The final attempt at negation, which is particularly interesting for us, because of its similarities to Routley's treatment of negation, singles out an arbitrary subset $U$ of the semi-lattice for $\mathbf{R}$ and an arbitrary subset $U$ of $S \times W$ for $\mathbf{E}$, and defines the valuation conditions for negation for $\mathbf{R}$ and $\mathbf{E}$ by the appropriate condition below. 
CRU $\quad X \vDash \sim \mathcal{A} \quad$ iff $\quad$ for all $Y$, either $Y \not \models \mathcal{A}$ or $X \cup Y \in U$;

CEU $X, w_{i} \vDash \mathcal{A}$ iff for all $Y$ and for all $w_{j}$ such that $w_{i} \leq w_{j}$ either $Y, w_{j} \not \models \mathcal{A}$ or $\left\langle X \cup Y, w_{j}\right\rangle \in U$.

The resulting semantics, however, invalidates some theorems of $\mathbf{R}$ and E such as $\mathcal{A} \vee \sim \mathcal{A}, \mathcal{A} \rightarrow \sim \sim \mathcal{A}$ and $\sim(\mathcal{A} \wedge \mathcal{B}) \rightarrow \sim \mathcal{A} \vee \sim \mathcal{B}$ (see [27, p. 166]).

\subsection{Positive Models}

Routley's models for $\mathbf{E}_{+}$are similar to Urquhart's with respect to the semilattice structure with possible worlds. One notable difference is the definition of validity, but this definition turns out to be equivalent. The other differences worth noting are the modifications to the semantics for $\mathbf{R}_{+}$.

Definition 2.3. A model for $\mathbf{E}_{+}$is a structure $\mathfrak{M}=\langle G, K, \leq, 0, N, v\rangle$ such that $\langle N, \cup, 0\rangle$ is a semi-lattice with lattice zero $0, K$ is a set of elements that includes $G, \leq$ is a reflexive and transitive relation on $K$, and $v$ is a valuation function such that for every atomic formula $p$, every element $\alpha \in N$, and every $H \in K$, either $v(p, \alpha, H)=T$ or $v(p, \alpha, H)=F$, but not both. We will simplify this notation to $v, H, \alpha \vDash p$ for $v(p, \alpha, H)=T$ and $v, H, \alpha \not \models p$ for $v(p, \alpha, H)=F$, typically dropping $v$. Finally, $v$ is extended to all formulas by the following conditions.

EP1 $H, \alpha \vDash \mathcal{A} \wedge \mathcal{B} \quad$ iff $\quad H, \alpha \vDash \mathcal{A}$ and $H, \alpha \vDash \mathcal{B}$

EP2 $H, \alpha \vDash \mathcal{A} \vee \mathcal{B} \quad$ iff $H, \alpha \vDash \mathcal{A}$ or $H, \alpha \vDash \mathcal{B}$

EP3 $H_{i}, \alpha \vDash \mathcal{A} \rightarrow \mathcal{B} \quad$ iff $\quad$ for every $H_{j} \in K$ and every $\beta \in N$ if $H_{i} \leq H_{j}$ and $H_{j}, \beta \vDash \mathcal{A}$, then $H_{j}, \alpha \cup \beta \vDash \mathcal{B}$

Routley, unlike Urquhart, included the world $G$ which may be thought of as either some designated world or the "actual world" in that model. A formula $\mathcal{A}$ is true in a model iff $G, 0 \vDash \mathcal{A}$, and valid iff true in every model. This definition is equivalent to Urquhart's. If a formula is not valid on Urquhart's models, then there is a world in the model at which the formula is not true (given the empty piece of information). To this model there corresponds a Routley-model with that world being named $G$ at which, given 0 , the formula is not true. The other direction is trivial.

To construct models for $\mathbf{R}_{+}$, Routley adds the following hereditariness requirement to the $\mathbf{E}_{+}$-models.

If $H_{i}, \alpha \vDash p$ and $H_{i} \leq H_{j}$, then $H_{j}, \alpha \vDash p$, for every atomic formula $p$, every $\alpha \in N$ and every $H_{i}, H_{j} \in K$. 
This requirement can be shown to extend to all formulas by a simple proof.

Thus, a major difference between Urquhart and Routley's positive models is their treatment of $\mathbf{R}$. It appears as if Urquhart had identified a minimal structure to capture (via soundness but not completeness) the positive fragment of $\mathbf{R}$, and made the necessary modifications to capture $\mathbf{E}_{+}$. In contrast, it appears that Routley's semantic system was first constructed for $\mathbf{E}_{+}$and then modified minimally to provide soundness (but, again, not completeness) for $\mathbf{R}_{+}$.

The manuscript contains a plethora of ideas concerning the treatment of negation. This is surprising given that for many logicians (including us) the most interesting part of the emergent joint Routley-Meyer semantics for relevance logic has to do with relevant implication and the ternary accessibility relation. Having briefly reviewed Routley's positive models, we now move on to the various attempts to model negation based on these positive models.

\subsection{Simplified Semantics for $\mathrm{R}$}

Routley's simplified semantics for $\mathbf{R}$ are constructed by simplifying his "Forced Negation Models." After producing his positive models, Routley's first (in order of appearance in the manuscript) idea for the treatment of negation is what he calls "Forced Negation Models." These models will be explicated in a later section. Here, however, the simplified semantics for $\mathbf{R}$ are considered. For the simplified models, the semi-lattice structure with lattice zero of the positive models is appended with an arbitrarily chosen subset of the semi-lattice, and an interesting pair of conditions. The simplification refers to a lack of what we might call possible worlds. The resulting structure is similar to Urquhart's semi-lattice semantics with two exceptions. First, Routley's condition for negation slightly differs from Urquhart's in that Routley's arbitrarily chosen set $P$ is the set theoretic complement of Urquhart's $U$. This will be shown in more detail below. The second is the additional requirements Routley employs in his attempt to capture the behavior of negation.

We leave the motivation and nature of the simplification to the section on "Forced Negation Models," where we will be able to consider both semantic systems simultaneously.

Definition 2.4. A simplified $\mathbf{R}$-model is a structure $\mathfrak{M}=\langle 0, N, \cup, P, v\rangle$ such that $N$ is a semi-lattice under $\cup$ with lattice zero $0, P$ is a set of elements of $N, v$ is a valuation function such that, for every $\alpha \in N$ and 
every atomic formula $p, v(p, \alpha)=T$ or $v(p, \alpha)=F$ (but not both), and the following conditions hold:

(i) If, for every $\beta \in N, P(\alpha \cup \beta)$ materially implies $P(\gamma \cup \beta)$, then $\alpha=\gamma$ (the simplified reduction requirement).

(ii) For every atomic $p$ and every $\alpha \in N$, if $v(p, \alpha)=F$ then for some $\beta \in N P(\alpha \cup \beta)$ (the simplified falsity requirement). [25, p. 22/14]

Again, let us employ our convenient notational shortcuts $\alpha \vDash \mathcal{A}$ and $\alpha \not \models \mathcal{A}$. The valuation function is extended as follows for the connectives $\wedge$, $\sim$ and $\rightarrow$ :

(1) $\alpha \vDash \mathcal{A} \wedge \mathcal{B} \quad$ iff $\quad \alpha \vDash \mathcal{A}$ and $\alpha \vDash \mathcal{B}$;

(2) $\alpha \vDash \mathcal{A} \rightarrow \mathcal{B} \quad$ iff $\quad$ for every $\beta \in N$, if $\beta \vDash \mathcal{A}$, then $\alpha \cup \beta \vDash \mathcal{B}$;

(3) $\alpha \vDash \sim \mathcal{A}$ iff for every $\beta \in N$, if $P(\alpha \cup \beta)$, then $\beta \not \models \mathcal{A}$.

Truth in a model is defined as expected: a formula $\mathcal{A}$ is true in a simplified $\mathbf{R}$-model when $0, v \vDash \mathcal{A}$. A wff $\mathcal{A}$ is valid for the simplified $\mathbf{R}$-models if and only if it is true in every simplified $\mathbf{R}$-model.

The difference between Routley and Urquhart's valuation conditions for negation are not significant. Given the same semi-lattice structure, we may define $P=S-U$. Doing so results in the equivalence between the valuation conditions, for Urquhart's condition is already equivalent to " $X \vDash \sim \mathcal{A}$ iff for all $Y, X \cup Y \notin U$ implies $Y \not \models A$." By replacing $S-U$ with $P$, Routley's condition results. Both conditions correspond to defining negation via $A \rightarrow$ $f$, but differ on whether to notationally privilege the elements of the semilattice corresponding to $f$ or to its set theoretic complement.

By selectively reducing the simplified $\mathbf{R}$-models to smaller sets of connectives, we can define semantics for various fragments of $\mathbf{R}$. As the semantics are identical to Urquhart's with the exception of the treatment of negation, the semantics for the positive fragments of $\mathbf{R}$ are sound for their respective fragments. Furthermore, a completeness proof is possible for the implicational fragment along the lines of Urquhart's proof in [27]. Given the similarities in the treatment of negation, the following lemma also follows.

Lemma 2.5. All instances of the axiom schemas $(\mathcal{A} \rightarrow \sim \mathcal{A}) \rightarrow \sim \mathcal{A}$ and $(\mathcal{A} \rightarrow \sim \mathcal{B}) \rightarrow(\mathcal{B} \rightarrow \sim \mathcal{A})$ are valid in the simplified $\mathbf{R}$-models.

Proof. One should be fairly convinced that the similarities to Urquhart's semi-lattice semantics with the exception of negation ensure that the axioms 
that do not contain disjunction or negation are valid. The interesting cases are those of negation, since Routley's semantics includes the falsity and reduction requirements. We shall demonstrate that axioms (A14)-(A15) of $\mathbf{R}$ are valid.

1. Axiom $(\mathcal{A} \rightarrow \sim \mathcal{A}) \rightarrow \sim \mathcal{A}$.

Assume for reductio that there is a simplified model wherein $0 \not \models(\mathcal{A} \rightarrow$ $\sim \mathcal{A}) \rightarrow \sim \mathcal{A}$. It follows from the truth condition for implication that there is a $\beta$ such that $\beta \vDash \mathcal{A} \rightarrow \sim \mathcal{A}$, but $\beta \not \models \sim \mathcal{A}$ (for $\beta \cup 0=\beta$ ). From the latter, we can infer that there is a $\gamma$ such that $\gamma \vDash \mathcal{A}$ and $P(\beta \cup \gamma)$. From $\beta \vDash \mathcal{A} \rightarrow \sim \mathcal{A}, \gamma \vDash \mathcal{A}$, and the truth condition for implication, it follows that $\beta \cup \gamma \vDash \sim \mathcal{A}$. Because of the properties of $\cup$, it follows from $P(\beta \cup \gamma)$ that $P((\beta \cup \gamma) \cup \gamma)$. From this last fact, the truth condition for negation, and $\beta \cup \gamma \vDash \sim \mathcal{A}$, it follows that $\gamma \not \models \mathcal{A}$, which contradicts our first inferred fact about $\gamma$.

2. Axiom $(\mathcal{A} \rightarrow \sim \mathcal{B}) \rightarrow(\mathcal{B} \rightarrow \sim \mathcal{A})$.

Assume for reductio that $0 \not \models((\mathcal{A} \rightarrow \sim \mathcal{B}) \rightarrow(\mathcal{B} \rightarrow \sim \mathcal{A}))$. From the truth condition for implication, we get that there is an $\alpha$ such that $\alpha \vDash(\mathcal{A} \rightarrow \sim \mathcal{B})$, but $\alpha \not \models(\mathcal{B} \rightarrow \sim \mathcal{A})$. From the latter, it follows that there is a $\beta$ such that $\beta \vDash \mathcal{B}$, but $\alpha \cup \beta \not \models \sim \mathcal{A}$. From the latter, it follows that there is a $\gamma$ such that $P((\alpha \cup \beta) \cup \gamma)$ and $\gamma \vDash \mathcal{A}$. However, given $\alpha \vDash(\mathcal{A} \rightarrow \sim \mathcal{B}), \gamma \vDash \mathcal{A}$ and the truth condition for implication, we can infer that $\alpha \cup \gamma \vDash \sim \mathcal{B}$. From this last fact, the truth condition for negation, and $P((\alpha \cup \gamma) \cup \beta)$ it follows that $\beta \not \models \mathcal{B}$, which contradicts the first inferred fact about $\beta$.

The falsity and reduction requirements are not used in the above proof. Given the falsity and reduction requirements, it might be conjectured that Routley's semantics is sound for $\mathbf{R}$. Unfortunately, a counter-model to the last negation axiom $\sim \sim \mathcal{A} \rightarrow \mathcal{A}$ is possible. To construct a counter-model for this axiom, first we need to construct a semi-lattice semantics that adheres to the conditions set out by Routley.

One possible simplified $\mathbf{R}$-model is the infinite join-semi-lattice $\mathfrak{N}_{\infty}$ in Figure 1 - with any valuation for the atomic formulas — as we explain below.

1. We note that the labeling on the left indicates that all the elements on the level are in $P$ or are not in $P$ (when the label is $\neg P$ ). $P$ and $\neg P$ continue to alternate between the levels. Given this, (ii) from Definition 2.4 is obviously satisfied - no matter what the valuation is - because for any element $\alpha$ there is another element $\beta$ such that $\alpha \leq \beta$ and $P(\beta)$, hence, $P(\alpha \cup \beta)$ too. 


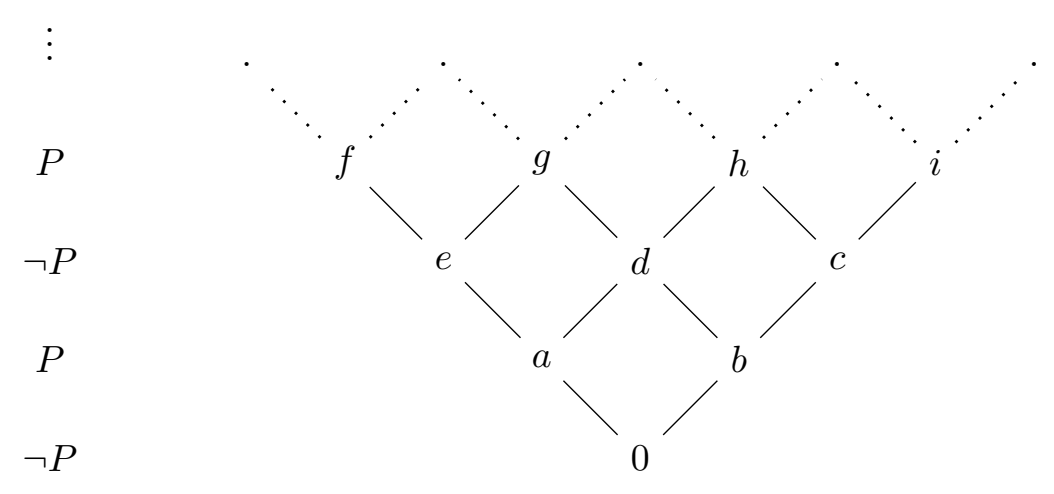

Figure 1: The infinite diamond $\mathfrak{N}_{\infty}$

2. Now we show that (i) is also true. Our strategy is to show that if a pair of elements $\alpha, \gamma$ are distinct in the semi-lattice (as suggested by the Hasse diagram in Figure 1), then there is an element $\beta$ with $P(\alpha \cup \beta)$ true but $P(\gamma \cup \beta)$ false. We consider two cases depending on whether $x$ and $y$ are comparable with respect to $\leq$ or not.

2.1 Let $x$ and $y$ be incomparable, that is, neither $x \leq y$ nor $y \leq x$ holds. Although $x$ and $y$ may not be on the same level, if $x \cup y$ is on level $n+1$ and $P(x \cup y)$, then there are elements $x^{\prime}$ and $y^{\prime}$ that are on level $n, x^{\prime} \cup y^{\prime}=x \cup y$ and $x \leq x^{\prime}$ and $y \leq y^{\prime}$. This means that we may concentrate on the latter pair of elements (which are like $d$ and $c$ in the diagram). We note that $x^{\prime}$ and $y^{\prime}$ are also incomparable, and each has another element (beyond $x^{\prime} \cup y^{\prime}$ ) immediately above it that is in $P$. Let us assume that these are $x^{\prime \prime}$ and $y^{\prime \prime}$. Then, $P\left(x \cup y^{\prime \prime}\right)$ is false, while $P\left(x \cup x^{\prime \prime}\right)$ is true; switching $x$ and $y$ throughout $P\left(y \cup x^{\prime \prime}\right)$ is false and $P\left(y \cup y^{\prime \prime}\right)$ holds. This shows that $x$ and $y$ are distinct under the simplified reduction principle. This argument appeals only to properties of join elements, and the fact that each element has two elements immediately above it, which implies that if $P(x \cup y)$ is false, then a permutation of $x^{\prime \prime}$ and $y^{\prime \prime}$ yields the desired conclusion.

2.2 Let us assume that $x$ and $y$ are comparable, but distinct. Then, without loss of generality, we can take $x \leq y$ to be the case. Again, there is a symmetry between $P$ and $\neg P$, hence, we only consider the former in detail.

Let $x \cup y \in P$ and $y \in P$. Since the elements are distinct, $x \notin P$ may be the case. If the difference between $y$ and $x$ is more than one level $(\ell(x)+1<\ell(y))$, then there is an $x^{\prime}$ immediately below $y$. Let $x \leq x^{\prime} \leq y$ be the case, where $\ell\left(x^{\prime}\right)+1=\ell(y)$, hence, $x^{\prime} \notin P$. Then $P\left(y \cup x^{\prime}\right)$ but $P\left(x \cup x^{\prime}\right)$ fails. By the construction of the semi-lattice, there is a $y^{\prime}$ such 
that $x^{\prime} \leq y^{\prime}, \ell(y)=\ell\left(y^{\prime}\right)$ but $y \neq y^{\prime}$. Then $P\left(x \cup y^{\prime}\right)$ but not $P\left(y \cup y^{\prime}\right)$. This completes our proof that $\mathfrak{N}_{\infty}$ is a simplified structure for $\mathbf{R}$.

3. The infinite semi-lattice $\mathfrak{N}_{\infty}$ above is a join-semi-lattice with lattice 0 . It satisfies the simplified reduction requirement, and the falsity requirement - as we showed above. To create a counter-model to $\sim \mathcal{A} \rightarrow \mathcal{A}$ using this semi-lattice, let $x \vDash q$ if $x \in P$ and $x \not \models q$ if $x \notin P$. As a result, $0 \not \models q$. However, it follows from the above stipulations that, for every element $z$ of the semi-lattice, $z \not \models \sim q$. That is, for every element $z$, there is an element $w$ such that $P(z \cup w), z \cup w=w$ and $w \vDash q$. This is ensured by the fact that there is always a "higher" element in the semi-lattice in the set $P$, and the fact that elements in the set $P$ make $q$ true (i.e., for all $w \in P$, $w \vDash q$ ). It follows from the fact that every element $z$ is such that $z \not \models \sim q$ that $0 \vDash \sim \sim q$. Given that both $0 \vDash \sim \sim q$ and $0 \not \models q$, this simplified R-model is a counter-example to the axiom $\sim \mathcal{A} \rightarrow \mathcal{A}$, which demonstrates that Routley's simplified $\mathbf{R}$-models are not models of $\mathbf{R}$.

Overall, the implicational fragment $\mathbf{R}_{\rightarrow}$ is sound and complete for Routley's simplified semantics, and the positive fragment $\mathbf{R}_{+}$is sound for it. The falsity and reduction requirements were insufficient to produce models for R. Thus, we may add Routley's attempt at negation to Urquhart's list of failed attempts at treating negation in the semi-lattice semantics of $\mathbf{R}$.

\subsection{Forced Negation Models}

Routley's "Forced Negation Models" take the rich framework of the positive models and, in an attempt to model negation, add a relation between elements of the semi-lattice and what can be called possible worlds. This relation is used in the treatment of negation. Again, we will conveniently write $H_{i}, \alpha \vDash \mathcal{A}$ and $H_{i}, \alpha \not \models \mathcal{A}$ in place of Routley's $v\left(\mathcal{A}, \alpha, H_{i}\right)=T$ and $v\left(\mathcal{A}, \alpha, H_{i}\right)=F$, respectively. According to Routley, the valuation condition for disjunction on these models is quite interesting, although the proof of this fact supposedly contains an erroneous use of the reduction requirement for these models.

Definition 2.6. A forced negation model for $\mathbf{R}$ is a structure $\mathfrak{M}=\langle G, K, \leq$, $0, N, P, v\rangle$, where $K$ is a set of elements including $G, \leq$ is a reflexive and transitive relation on $K,\langle N, 0\rangle$ is a semi-lattice with join operation denoted by $\cup$, and with lattice zero $0 . v$ is a function such that for every atomic $p$ and every $\alpha \in N$ and $H_{i} \in K$, either $H_{i}, \alpha \vDash p$ or $H_{i}, \alpha \not \models p$, and $P \subseteq N \times K$ such that the following requirements are satisfied:

1 If, for every $\beta \in N$ and $H \in K, H_{1} \leq H$ and $P(\alpha \cup \beta, H)$ imply 
$H \leq H_{2}$ and $P\left(\gamma \cup \beta, H_{2}\right)$, then $\left(\alpha, H_{1}\right)=\left(\gamma, H_{2}\right) ;$

2 if $H_{1}, \alpha \vDash p$ and $H_{1} \leq H_{2}$, then $H_{2}, \alpha \vDash p$, for every atomic formula $p$, every $\alpha \in N$ and every $H_{1}, H_{2} \in K$;

3 for every atomic $p$, every $\alpha \in N$ and every $H_{1} \in K$, if $H_{1}, \alpha \not \models p$, then for some $\beta$ and $H_{2}$ both $H_{1} \leq H_{2}$ and $P\left(\alpha \cup \beta, H_{2}\right)$.

The requirements are named, respectively, the reduction requirement (1), the hereditariness requirement (2), and the falsity requirement (3). Finally, the valuation $v$ is extended to $\wedge, \rightarrow$, and $\sim$ as follows:

FN1 $\quad H, \alpha \vDash \mathcal{A} \wedge \mathcal{B} \quad$ iff $\quad H, \alpha \vDash \mathcal{A}$ and $H, \alpha \vDash \mathcal{B}$;

FN2 $H_{i}, \alpha \vDash \mathcal{A} \rightarrow \mathcal{B}$ iff for every $H_{j} \in K$ and $\beta \in N$, if $H_{i} \leq H_{j}$ and $H_{j}, \beta \vDash \mathcal{A}$ then $H_{j}, \alpha \cup \beta \vDash \mathcal{B}$;

FN3 $H_{i}, \alpha \vDash \sim \mathcal{A}$ iff for every $H_{j} \in K$ and $\beta \in N$, if $H_{i} \leq H_{j}$ and $P\left(\alpha \cup \beta, H_{j}\right)$ then $H_{j}, \beta \not \models \mathcal{A}$.

A formula $\mathcal{A}$ is true in the forced negation model $\mathfrak{M}$ iff $G, 0 \vDash \mathcal{A}$. A formula is (forced-negation-) valid iff it is true in every forced negation model.

In these models, Routley treats disjunction as a defined connective via its usual definition using conjunction and negation. The valuation condition stated by Routley for disjunction is not what one would expect. The statement of the condition is split into two. The first is not very surprising.

FN4.1 If $H, \alpha \vDash \mathcal{A}$ or $H, \alpha \vDash \mathcal{B}$, then $H, \alpha \vDash \mathcal{A} \vee \mathcal{B}$.

However, the second half includes an unexpected proviso.

FN4.2 If $H, \alpha \vDash \mathcal{A} \vee \mathcal{B}$, then $H, \alpha \vDash \mathcal{A}$ or $H, \alpha \vDash \mathcal{B}$, provided that for some formula $\mathcal{C}$ and some $\beta \in N, H, \alpha \cup \beta \not \models \mathcal{C}$.

This surprising proviso allows for an application of the falsity requirement in the proof; however, the reduction requirement is incorrectly applied in the proof attempt. This pair of valuation conditions for defined disjunction has not been proven to work. In fact, it appears unlikely such a proof is possible.

Routley's simplified models for $\mathbf{R}$ are constructed by modifying the forced negation models to eliminate the possible worlds. However, at the end of the manuscript, in his "Covering Note," Routley points out that 
All of my "proofs" that simplified models will work for $\mathrm{R}$ have broken down. [24, p. 95/69]

However, Routley does not indicate where or why the so-called "proofs" break down. In Section 2.3, the simplified models are shown by us to be unsound for the logic $\mathbf{R}$. In particular, $\sim \sim \mathcal{A} \rightarrow \mathcal{A}$ is not valid on the simplified models.

Routley uses a key lemma to demonstrate the redundancy of the possible worlds and motivate a move from the forced negation models to the simplified models for $\mathbf{R}$. Routley provides a brief sketch of this proof in which he claims that both the falsity and reduction requirements are used essentially. In this lemma, he states, among another things, that on the forced negation models:

For every $H \in K, v(A, \alpha, H)=v(\sim \sim A, \alpha, H)$.

That is, $H, \alpha \vDash \mathcal{A}$ iff $H, \alpha \vDash \sim \sim \mathcal{A}$. However, in our proof of the inadequacy of the simplified models, the counter-model to $\sim \sim \mathcal{A} \rightarrow \mathcal{A}$ shows that $\alpha \vDash \mathcal{A}$ iff $\alpha \vDash \sim \sim \mathcal{A}$ is not the case for the simplified models as defined.

This leaves open two possibilities. Either Routley's error lies in this motivating lemma, or it lies in the forced negation models themselves. As will be demonstrated below, Routley's forced negation models fail to validate $\sim \sim \mathcal{A} \rightarrow \mathcal{A}$. The counter-example is similar to the model we used to demonstrate the inadequacy of the simplified models, for it uses the same semi-lattice structure.

Routley's forced negation models do not validate $\sim \sim \mathcal{A} \rightarrow \mathcal{A}$. The counter-example we will consider merely adds a single possible world, $G$, to the counter-model for the simplified models in Section 2.3, with the appropriate changes to deal with such an addition.

Consider the infinite semi-lattice $\mathfrak{N}_{\infty}$ in Figure 1 . Let $K$ contain only the member $G$, and let $P(\alpha, G)$ iff $\alpha$ is an element of the semi-lattice labeled as $P$. The hereditariness requirement is trivially satisfied. To see that the reduction and falsity requirements are satisfied, the reader is encouraged to modify the relevant proofs in Section 2.3 with the addition of the single world $G$.

Finally, let $G, \alpha \vDash q$ if $G, \alpha \in P$ and $G, \alpha \not \models q$ if $G, \alpha \notin P$. As a result, $G, 0 \not \models q$. However, it follows from the above stipulations that, for every element $\beta$ of the semi-lattice, $G, \beta \not \models \sim q$, because there is always another element $\gamma$ such that $P(\beta \cup \gamma, G)$ and $G, \gamma \vDash q$. This is ensured by the fact that there is always a 'higher' element in the semi-lattice in the set $P$, and 
the fact that elements in the set $P$ make $q$ true. It follows from the fact that every element $\beta$ is such that $G, \beta \not \models \sim q$ that $G, 0 \vDash \sim \sim q$.

Given that both $G, 0 \vDash \sim \sim q$ and $G, 0 \not \models q$, this forced negation model is a counter-example to the axiom $\sim \sim \mathcal{A} \rightarrow \mathcal{A}$, which demonstrates that the forced negation models are unsound for $\mathbf{R}$, and indeed every logic with this axiom.

Thus, Routley's error lies in the forced negation models, wherein $\sim \sim \mathcal{A} \rightarrow$ $\mathcal{A}$ is invalid. This is somewhat to be expected, as the treatment of negation in the models is similar to defined $\sim \mathcal{A}$ as $\mathcal{A} \rightarrow f$, a fact, which Routley highlighted. He claimed that defining negation via $f$ and $f$ via " $H, \alpha \not \models f$ iff $P(\alpha, H)$ " [24]. In other words, the pairs in $P$ could be thought of as consistent in the respect that they do not make the falsity constant $f$ true.

Given that neither the simplified models nor the forced negation models validate $\sim \sim \mathcal{A} \rightarrow \mathcal{A}$, we are left to wonder why Routley recognized the failure of his "proofs" with respect to the simplified models, but not the forced negation models. However, Routley's ideas concerning the forced negation models are illuminating. The unexpected valuation condition for disjunction appears to indicate that Routley anticipated problems with the semantics for fragments of $\mathbf{R}$ that include disjunction. Additionally, the reduction and falsity requirements are used in this section sparingly. Both are used in the failed proof for the valuation condition for disjunction, and the reduction requirement is suggested to be used non-essentially in a proof that the falsity requirement applies to all formulas. Finally, both requirements are cited in the failed "proof" motivating the simplified semantics.

\subsection{Direct Negation Models}

Another of Routley's ideas concerning the treatment of negation is what he called the "Direct Negation Models." In these models we find the same semi-lattice structure with lattice zero. However, the possible-worlds are enriched to produce models for negation. Two novel ideas are combined in these models. The first is the introduction of worlds at which implicational formulas are given arbitrary truth values. We could call these non-normal worlds, the abnormality following only from their valuation of conditionals. While Routley does not use the term "non-normal," we will do so here for easy reference. The second idea is the pairing of each possible world with a unique non-normal world. By combining these two ideas, the models that result are sound for the logic $\mathbf{E}$.

Definition 2.7. A direct negation model for $\mathbf{E}$ is a structure $\mathfrak{M}=\langle G, K, \leq$, 
$0, N, \cup, v\rangle$, where as before, $\langle N, \cup, 0\rangle$ is a semi-lattice with lattice zero $0, K$ is a set of elements, $G \in K$, and following conditions hold:

(1) For each $H_{i} \in K$, there is a unique $J_{i} \in K$;

(2) $\leq$ is a transitive and reflexive relation on $M=\left\{H_{i}: h_{i} \in K\right\}$;

(3) $G=H_{0}$ (i.e., $\left.G \in M\right)$;

(4) for every $\alpha \in N$ and $H_{i}$ and $J_{i} \in K, v$ assigns either $T$ or $F$ to each atomic proposition (as before);

(5) for every $\alpha \in N$ and $J_{i} \in K, v$ assigns either $T$ or $F$ to each implicational formula.

We will keep to Routley's notation and use $I, I_{1}, I_{2}, \ldots I_{n}, \ldots$ as variables ranging over the elements of $K$.

Definition 2.8. The valuation $v$ is extended as follows:

DN1 $I, \alpha \vDash \mathcal{A} \wedge \mathcal{B} \quad$ iff $\quad I, \alpha \vDash \mathcal{A}$ and $I, \alpha \vDash \mathcal{B}$;

DN2 $I, \alpha \vDash \mathcal{A} \vee \mathcal{B} \quad$ iff $\quad I, \alpha \vDash \mathcal{A}$ or $I, \alpha \vDash \mathcal{B}$;

DN3 $H_{i}, \alpha \vDash \sim \mathcal{A} \quad$ iff $\quad J_{i}, \alpha \not \models \mathcal{A}$;

DN4 $J_{i}, \alpha \vDash \sim \mathcal{A} \quad$ iff $\quad H_{i}, \alpha \not \models \mathcal{A}$;

DN5.1 if $H_{i}, \alpha \vDash \mathcal{A} \rightarrow \mathcal{B}$, then for every $\beta \in N$ and $H_{j} \in K$, if $H_{i} \leq H_{j}$, then

(i) if $H_{j}, \beta \vDash \mathcal{A}$ then $H_{j}, \alpha \cup \beta \vDash \mathcal{B}$, and

(ii) if $J_{j}, \alpha \cup \beta \vDash \mathcal{A}$ then $J_{j}, \beta \vDash \mathcal{B}$;

DN5.2 if $H_{i}, \alpha \vDash \mathcal{A} \rightarrow \mathcal{B}$ and $H_{i}, \alpha, \not \models \mathcal{B}$, then $H_{i}, \alpha \not \models \mathcal{A}$;

DN6 if $H_{i}, \alpha \not \models \mathcal{A} \rightarrow \mathcal{B}$, then for some $\beta \in N$ and some $H_{j} \in K$, $H_{i} \leq H_{j}$ and $H_{j}, \beta \vDash \mathcal{A}$ and $H_{j}, \alpha \cup \beta \not \models \mathcal{B}$, and $J_{j}, \alpha \cup \beta \vDash \mathcal{A}$ and $J_{j}, \beta \not \models \mathcal{B}$.

The condition DN5.2 is intended to replace the reduction requirement, and DN5.1 is intended to capture contraposition principles. The condition DN6 results from simplifying Routley's original condition in a way suggested 
in the manuscript. (Originally, the final clause used a bound $\gamma$ instead of $\beta.)^{13}$

A formula $\mathcal{A}$ is true in a model if and only if $G, 0 \vDash \mathcal{A}$. A formula is valid when it is true in all direct negation models.

To create models for the logic $\mathbf{R}$, the following two modifications must be made.

$1 \leq$ is extended from $M$ to all of $K$ by setting $J_{1} \leq J_{2}$ iff $H_{2} \leq H_{1}$.

2 If $I_{i}, \alpha \vDash \mathcal{A}$ and $I_{i} \leq I_{j}$, then $I_{j}, \alpha \vDash \mathcal{A}$ for every atomic formula for every $\alpha$ and $I_{i}, I_{j} \in K$ and for every entailment for every $\alpha$ and $J_{i}, J_{j} \in K$.

This second requirement, the hereditariness requirement, can be shown to extend to all formulas $\mathcal{A}$, having been defined here for every initial case. Again, truth and validity are defined as expected.

Interestingly, the axioms for negation for $\mathbf{E}$, and thus also for $\mathbf{R}$, are valid on these direct negation models.

Lemma 2.9. The axioms $(\mathcal{A} \rightarrow \sim \mathcal{A}) \rightarrow \sim \mathcal{A}$ and $(\mathcal{A} \rightarrow \sim \mathcal{B}) \rightarrow(\mathcal{B} \rightarrow \sim \mathcal{A})$, and $\sim \sim \mathcal{A} \rightarrow \mathcal{A}$ are valid on the direct negation models.

Proof. 1. Axiom $(\mathcal{A} \rightarrow \sim \mathcal{A}) \rightarrow \sim \mathcal{A}$.

Assume for reductio that there is a directed negation model wherein $G, 0 \not \models$ $(\mathcal{A} \rightarrow \sim \mathcal{A}) \rightarrow \sim \mathcal{A}$. From condition DN6, it follows that there is an $\alpha \in N$ and $H_{i} \in K$ such that $G \leq H_{i}$ and $H_{i}, \alpha \vDash \mathcal{A} \rightarrow \sim \mathcal{A}, H_{i}, \alpha \not \models \sim \mathcal{A}, J_{i}, \alpha \vDash$ $\mathcal{A} \rightarrow \sim \mathcal{A}$, and finally $J_{i}, \alpha \not \models \sim \mathcal{A}$. From the last fact and DN4, it follows that $H_{i}, \alpha \vDash \mathcal{A}$. However, it follows from $H_{i}, \alpha \vDash \mathcal{A} \rightarrow \sim \mathcal{A}$ and $H_{i}, \alpha \not \models \sim \mathcal{A}$ that $H_{i}, \alpha \not \models \mathcal{A}$, which gives us our required contradiction.

2. Axiom $(\mathcal{A} \rightarrow \sim \mathcal{B}) \rightarrow(\mathcal{B} \rightarrow \sim \mathcal{A})$.

Suppose for reductio that for some model we have $G, 0 \not \models(\mathcal{A} \rightarrow \sim \mathcal{B}) \rightarrow$ $(\mathcal{B} \rightarrow \sim \mathcal{A})$. It follows that there is an $\alpha$ and $H_{i}$ such that $G \leq H_{i}, H_{i}, \alpha \vDash$ $(\mathcal{A} \rightarrow \sim \mathcal{B})$, and $H_{i}, \alpha \not \models(\mathcal{B} \rightarrow \sim \mathcal{A})$. From $H_{i}, \alpha \not \models(\mathcal{B} \rightarrow \sim \mathcal{A})$ and DN6 we get that there is a $\beta$ and $H_{j}$ such that $H_{i} \leq H_{j}, H_{j}, \beta \vDash \mathcal{B}, H_{j}, \alpha \cup \beta \not \models \sim \mathcal{A}$, $J_{j}, \alpha \cup \beta \vDash \mathcal{B}$, and $J_{j}, \beta \not \models \sim \mathcal{A}$. From $H_{j}, \alpha \cup \beta \not \models \sim \mathcal{A}$ and DN3 it follows that $J_{j}, \alpha \cup \beta \vDash \mathcal{A}$. With $H_{i}, \alpha \vDash(\mathcal{B} \rightarrow \sim \mathcal{A})$ and $H_{i} \leq H_{j}$ and $J_{j}, \alpha \cup \beta \vDash \mathcal{A}$, it follows by DN5.2 that $J_{j}, \beta \vDash \sim \mathcal{B}$. From this last fact and DN4 we get $H_{j}, \beta \not \models \mathcal{B}$, which gives us our required contradiction.

\footnotetext{
${ }^{13}$ One of us (NF) may have done more simplifying here than suggested by Routley. However, such simplification appears necessary for the proofs below. The last part of DN6 before simplification uses $J_{k}$ and $\gamma$ instead of $J_{j}$ and $\beta$.
} 
3. Axiom $\sim \mathcal{A} \rightarrow \mathcal{A}$.

Suppose for reductio that $G, 0 \not \models \sim \sim \mathcal{A} \rightarrow \mathcal{A}$. It follows that there is a $\beta$ and $H_{j}$ such that $G \leq H_{j}, H_{j}, \beta \vDash \sim \sim \mathcal{A}$, but $H_{j}, \beta \not \models \mathcal{A}$. By DN3 and $H_{j}, \beta \vDash \sim \sim \mathcal{A}$, we get that $J_{j}, \beta \not \models \sim \mathcal{A}$. From this last fact and $\mathrm{DN}$ it follows that $H_{j}, \beta \vDash \mathcal{A}$, which gives us our required contradiction.

Theorem 2.10. The logic $\mathbf{E}$ is sound for the direct negation models.

Proof. Axioms (A14)-(A16) are covered by the previous lemma. The proof for the remaining axioms is quite lengthy. However, it makes no use of either the $J$-worlds or the arbitrary valuations of entailments at $J$-worlds.

1. Axiom $\mathcal{A} \rightarrow \mathcal{A}$.

Suppose for reductio that $G, 0 \not \models \mathcal{A} \rightarrow \mathcal{A}$. Then there is a $\beta$ and $H_{i}$ such that $H_{i}, \beta \vDash \mathcal{A}$ and $H_{i}, \beta \not \models \mathcal{A}$, a contradiction.

2. $\operatorname{Axiom}(\mathcal{A} \rightarrow \mathcal{B}) \rightarrow((\mathcal{C} \rightarrow \mathcal{A}) \rightarrow(\mathcal{C} \rightarrow \mathcal{B}))$.

Suppose for reductio that $G, 0 \not \models(\mathcal{A} \rightarrow \mathcal{B}) \rightarrow((\mathcal{C} \rightarrow \mathcal{A}) \rightarrow(\mathcal{C} \rightarrow \mathcal{B}))$. It follows that there is an $\alpha$ and $H_{i}$ such that $G \leq H_{i}$ and $H_{i}, \alpha \vDash \mathcal{A} \rightarrow \mathcal{B}$ and $H_{i}, \alpha \not \models(\mathcal{C} \rightarrow \mathcal{A}) \rightarrow(\mathcal{C} \rightarrow \mathcal{B})$. From the latter it follows that there is a $\beta$ and $H_{j}$ such that $H_{i} \leq H_{j}$ and $H_{j}, \beta \vDash \mathcal{C} \rightarrow \mathcal{A}$, but $H_{j}, \alpha \cup \beta \not \models \mathcal{C} \rightarrow \mathcal{B}$. From the latter again we get that there is $\gamma$ and $H_{k}$ such that $H_{j} \leq H_{k}$, $H_{k}, \gamma \vDash \mathcal{C}$, and $H_{k}, \alpha \cup \beta \cup \gamma \not \models \mathcal{B}$. Using DN5.1, $H_{j}, \beta \vDash \mathcal{C} \rightarrow \mathcal{A}, H_{j} \leq H_{k}$, and $H_{k}, \gamma \vDash \mathcal{C}$, it follows that $H_{k}, \beta \cup \gamma \vDash \mathcal{A}$. By a similar argument from $H_{i}, \alpha \vDash \mathcal{A} \rightarrow \mathcal{B}$ and the transitivity of $\leq$, it follows that $H_{k}, \alpha \cup \beta \cup \gamma \vDash \mathcal{B}$, which gives us our contradiction.

3. Axiom $(\mathcal{A} \rightarrow(\mathcal{A} \rightarrow \mathcal{B})) \rightarrow(\mathcal{A} \rightarrow \mathcal{B})$.

Suppose for reductio that $G, 0 \not \models(\mathcal{A} \rightarrow(\mathcal{A} \rightarrow \mathcal{B})) \rightarrow(\mathcal{A} \rightarrow \mathcal{B})$. Then there is an $\alpha$ and $H_{i}$ such that $G \leq H_{i}, H_{i}, \alpha \vDash \mathcal{A} \rightarrow(\mathcal{A} \rightarrow \mathcal{B})$, but $H_{i}, \alpha \not \models \mathcal{A} \rightarrow \mathcal{B}$. From the latter we get that there is a $\beta$ and $H_{j}$ such that $H_{i} \leq H_{j}, H_{j}, \beta \vDash \mathcal{A}$, and $H_{j}, \alpha \cup \beta \not \models \mathcal{B}$. From $H_{j}, \beta \vDash \mathcal{A}, H_{i}, \alpha \vDash \mathcal{A} \rightarrow(\mathcal{A} \rightarrow \mathcal{B})$, and $H_{i} \leq H_{j}$ it follows that $H_{j}, \alpha \cup \beta \vDash \mathcal{A} \rightarrow \mathcal{B}$. The last fact, the reflexivity of $\leq$ and idempotence of $\cup$ and $H_{j}, \beta \vDash \mathcal{A}$ with DN5.1 imply that $H_{j}, \alpha \cup \beta \vDash \mathcal{B}$, which gives us our contradiction.

4. Axiom $(\mathcal{A} \rightarrow((\mathcal{B} \rightarrow \mathcal{D}) \rightarrow \mathcal{C})) \rightarrow((\mathcal{B} \rightarrow \mathcal{D}) \rightarrow(\mathcal{A} \rightarrow \mathcal{C}))$.

Suppose for reductio that $G, 0 \not \models(\mathcal{A} \rightarrow((\mathcal{B} \rightarrow \mathcal{D}) \rightarrow \mathcal{C})) \rightarrow((\mathcal{B} \rightarrow \mathcal{D}) \rightarrow$ $(\mathcal{A} \rightarrow \mathcal{C}))$. Then there is an $\alpha$ and $H_{i}$ such that $G \leq H_{i}$, and $H_{i}, \alpha \vDash$ $\mathcal{A} \rightarrow((\mathcal{B} \rightarrow \mathcal{D}) \rightarrow \mathcal{C})$ but $H_{i}, \alpha \not \models(\mathcal{B} \rightarrow \mathcal{D}) \rightarrow(\mathcal{A} \rightarrow \mathcal{C})$. From the latter, there is a $\beta$ and $H_{j}$ such that $H_{i} \leq H_{j}, H_{j}, \beta \vDash \mathcal{B} \rightarrow \mathcal{D}$, and $H_{j}, \alpha \cup \beta \not \models \mathcal{A} \rightarrow \mathcal{C}$. From the latter, it follows that there is a $\gamma$ and $H_{k}$ such that $H_{j} \leq H_{k}, H_{k}, \gamma \vDash \mathcal{A}$, and $H_{k}, \gamma \cup \alpha \cup \beta \not \models \mathcal{C}$. From $H_{k}, \gamma \vDash \mathcal{A}$, the transitivity of $\leq$ and $H_{i}, \alpha \vDash \mathcal{A} \rightarrow((\mathcal{B} \rightarrow \mathcal{D}) \rightarrow \mathcal{C})$, it follows by DN5.1 
that $H_{k}, \alpha \cup \gamma \vDash(\mathcal{B} \rightarrow \mathcal{D}) \rightarrow \mathcal{C}$. Similarly, applying DN5.1 again we get that $H_{k}, \alpha \cup \beta \cup \gamma \vDash \mathcal{C}$, which gives us our desired contradiction.

5. Axiom $(\mathcal{A} \wedge \mathcal{B}) \rightarrow \mathcal{A}$.

Suppose for reductio that $G, 0 \not \models(\mathcal{A} \wedge \mathcal{B}) \rightarrow \mathcal{A}$. Then there is an $\alpha$ and $H_{i}$ such that $G \leq H_{i}, H_{i}, \alpha \vDash \mathcal{A} \wedge \mathcal{B}$, and $H_{i}, \alpha \not \models \mathcal{A}$. However, from DN1 we get that $H_{i}, \alpha, \vDash \mathcal{A}$, a contradiction.

6. Axiom $(\mathcal{A} \wedge \mathcal{B}) \rightarrow \mathcal{B}$.

Similar to previous case.

7. Axiom $((\mathcal{C} \rightarrow \mathcal{A}) \wedge(\mathcal{C} \rightarrow \mathcal{B})) \rightarrow(\mathcal{C} \rightarrow(\mathcal{A} \wedge \mathcal{B}))$.

Suppose for reductio that $G, 0 \not \models((\mathcal{C} \rightarrow \mathcal{A}) \wedge(\mathcal{C} \rightarrow \mathcal{B})) \rightarrow(\mathcal{C} \rightarrow(\mathcal{A} \wedge \mathcal{B}))$. It follows that there is an $\alpha$ and $H_{i}$ such that $G \leq H_{i}, H_{i}, \alpha \vDash(\mathcal{C} \rightarrow \mathcal{A}) \wedge(\mathcal{C} \rightarrow$ $\mathcal{B})$, and $H_{i}, \alpha \not \models \mathcal{C} \rightarrow(\mathcal{A} \wedge \mathcal{B})$. From the latter we get that there is a $\beta$ and $H_{j}$ such that $H_{i} \leq H_{j}, H_{j}, \beta \vDash \mathcal{C}$, and $H_{j}, \alpha \cup \beta \not \models \mathcal{A} \wedge \mathcal{B}$. From DN1 we get that $H_{i}, \alpha \vDash \mathcal{C} \rightarrow \mathcal{A}$, and $H_{i}, \alpha \vDash \mathcal{C} \rightarrow \mathcal{B}$. Using DN5.1 on each of these with $H_{j}, \beta \vDash \mathcal{C}$, then using DN1 again, we arrive at $H_{j}, \alpha \cup \beta \vDash \mathcal{A} \wedge \mathcal{B}$, which gives us our contradiction.

8. Axiom $(((\mathcal{A} \rightarrow \mathcal{A}) \wedge(\mathcal{B} \rightarrow \mathcal{B})) \rightarrow \mathcal{C}) \rightarrow \mathcal{C}$.

Suppose for reductio that $G, 0 \not \models(((\mathcal{A} \rightarrow \mathcal{A}) \wedge(\mathcal{B} \rightarrow \mathcal{B})) \rightarrow \mathcal{C}) \rightarrow \mathcal{C}$. Then there is an $\alpha$ and $H_{i}$ such that $G \leq H_{i}, H_{i}, \alpha \vDash((\mathcal{A} \rightarrow \mathcal{A}) \wedge(\mathcal{B} \rightarrow \mathcal{B})) \rightarrow \mathcal{C}$, and $H_{i}, \alpha \not \models \mathcal{C}$. From $H_{i}, \alpha \vDash((\mathcal{A} \rightarrow \mathcal{A}) \wedge(\mathcal{B} \rightarrow \mathcal{B})) \rightarrow \mathcal{C}$, it follows that for every $\beta$ and $H_{j}$ such that $H_{i} \leq H_{j}$, if $H_{j}, \beta \vDash(\mathcal{A} \rightarrow \mathcal{A}) \wedge(\mathcal{B} \rightarrow \mathcal{B})$, then $H_{j}, \alpha \cup \beta \vDash \mathcal{C}$. If we let $\beta=0$ and $H_{j}=H_{i}$ (by the reflexivity of $\leq$ ), then we infer that $H_{i}, \alpha \vDash \mathcal{C}$, which gives us our contradiction.

9. Axiom $\mathcal{A} \rightarrow(\mathcal{A} \vee \mathcal{B})$

Suppose for reductio that $G, 0 \not \models \mathcal{A} \rightarrow(\mathcal{A} \vee \mathcal{B})$. Then there is an $\alpha$ and $H_{i}$ such that $G \leq H_{i}, H_{i}, \alpha \vDash \mathcal{A}$, and $H_{i}, \alpha \not \models \mathcal{A} \vee \mathcal{B}$. But then $H_{i}, \alpha \not \models \mathcal{A}$, giving us our contradiction.

10. Axiom $\mathcal{A} \rightarrow(\mathcal{B} \vee \mathcal{A})$.

Similar to previous case.

11. Axiom $((\mathcal{A} \rightarrow \mathcal{C}) \wedge(\mathcal{B} \rightarrow \mathcal{C})) \rightarrow((\mathcal{A} \vee \mathcal{B}) \rightarrow \mathcal{C})$

Suppose for reductio that $G, 0 \not \models((\mathcal{A} \rightarrow \mathcal{C}) \wedge(\mathcal{B} \rightarrow \mathcal{C})) \rightarrow((\mathcal{A} \vee \mathcal{B}) \rightarrow \mathcal{C})$ Then there is a $\beta$ and $H_{j}$ such that $0 \leq H_{j}, H_{j}, \beta \vDash((\mathcal{A} \rightarrow \mathcal{C}) \wedge(\mathcal{B} \rightarrow \mathcal{C}))$, but $H_{j}, \beta \not \models((\mathcal{A} \vee \mathcal{B}) \rightarrow \mathcal{C})$. From the latter, it follows that there is a $\gamma$ and $H_{k}$ such that $H_{j} \leq H_{k}, H_{k}, \gamma \vDash(\mathcal{A} \vee \mathcal{B})$, but $H_{k}, \gamma \cup \beta \not \models \mathcal{C}$. From $H_{k}, \gamma \vDash(\mathcal{A} \vee \mathcal{B})$, either $H_{k}, \gamma \vDash \mathcal{A}$ or $H_{k}, \gamma \vDash \mathcal{B}$. Suppose the former. Using $\mathrm{DN} 1$, it follows that $H_{j}, \beta \vDash(\mathcal{A} \rightarrow \mathcal{C})$. Using DN5.1, we may then infer that $H_{k}, \gamma \cup \beta \vDash \mathcal{C}$, thus deriving a contradiction.

12. Axiom $(\mathcal{A} \wedge(\mathcal{B} \vee \mathcal{C})) \rightarrow((\mathcal{A} \wedge \mathcal{B}) \vee(\mathcal{A} \wedge \mathcal{C}))$

Suppose for reductio that $G, 0 \not \models(\mathcal{A} \wedge(\mathcal{B} \vee \mathcal{C})) \rightarrow((\mathcal{A} \wedge \mathcal{B}) \vee(\mathcal{A} \wedge \mathcal{C}))$. Then there is a $\beta$ and $H_{i}$ such that $0 \leq H_{i}, H_{i}, \beta \vDash(\mathcal{A} \wedge(\mathcal{B} \vee \mathcal{C}))$, but 
$H_{i}, \beta \not \models((\mathcal{A} \wedge \mathcal{B}) \vee(\mathcal{A} \wedge \mathcal{C}))$. From the former, we get that $H_{i}, \beta \vDash \mathcal{A}$ and $H_{i}, \beta \vDash(\mathcal{B} \vee \mathcal{C})$. Thus, either $H_{i}, \beta \vDash \mathcal{B}$ or $H_{i}, \beta \vDash \mathcal{C}$. Whether the former or latter is the case, it follows that $H_{i}, \beta \vDash((\mathcal{A} \wedge \mathcal{B}) \vee(\mathcal{A} \wedge \mathcal{C}))$, which gives us a contradiction.

13. Rule (R1).

Assume that $G, 0 \vDash \mathcal{A} \rightarrow \mathcal{B}$ and that $G, 0 \vDash \mathcal{A}$. Given the reflexivity of 0 , it follows from DN5.1 that $G, 0 \vDash \mathcal{B}$.

14. Rule (R2).

Proof is trivial given DN1.

The direct negation models are, therefore, models for the logic $\mathbf{E}$.

The main work for modeling negation is done by the valuation conditions and the idea of pairing worlds together. The idea of arbitrarily assigning implication formulas arbitrary values at $J$-worlds appears to be doing work in preventing formulas such as replacement instances of $(\mathcal{A} \wedge \sim \mathcal{A}) \rightarrow \mathcal{B}$ from being valid. This is done by allowing that some $H$-worlds make both $\mathcal{A} \rightarrow \mathcal{A}$ and its negation true. The arbitrary assignments at $J$-worlds allow $H$-worlds to exist where an implication and its negation are given the value $T$ or $F$. Thus, the idea of arbitrary valuations for implicational formulas at $J$-worlds is to be used in the completeness proof, which ought to fail; the negation-free formula for which Urquhart's positive models fail to invalidate should also be valid on every direct negation model.

The idea of pairing worlds together to deal with negation can be found elsewhere in the semantics of relevance logic. For example, consider the Routley star operator, introduced by both Richard and Valerie Routley in [26]. In this paper, the Routley star operator is used to create set-ups in which either two-valued tautologies in $\wedge, \vee$, and $\sim$ fail to hold or contradictions are made to hold. The valuation condition for negation is defined by $\sim \mathcal{A}$ to be in the set up $H$ if and only if $\mathcal{A}$ is not in $H^{*} .{ }^{14}$ The main differences between the treatment of negation in the direct negation models and with Routley star operator are the uniform treatment of the set-ups by Routley and Routley, the difference between a possible world and a set-up in terms of the Routley star, and the treatment of the distinguished world $G$. For Routley and Routley, there is no guarantee in the models that $H=H^{*}$; however, when $H=H^{*}$, they call the set-up $H$ a world due to its completeness and consistency. Further, it is required that $G$ is a world (i.e., $G=G^{*}$ )

\footnotetext{
${ }^{14}$ The operational treatment of De Morgan negation in a distributive lattice - due to A. Białynicki-Birula and H. Rasiowa from 1957 - was known to some relevance logicians already. See Dunn [6] and [8, §4.3].
}

Australasian Journal of Logic (15:2) 2018, Article no. 3.2 
[26, p. 347]. Finally, Routley and Routley do not separate the set-ups into two classes, unlike the direct negation models.

Another, similar treatment of negation can be found in Restall [23], wherein four-valued worlds are similarly paired to deal with negation. At least, that is, the pairing of worlds is used essentially for the treatment of negated conditionals.

\section{From the desk of Robert K. Meyer}

Meyer had seen Routley's manuscript as well as Urquhart's draft for his paper [27]. However, Routley's manuscript mentions - somewhat disparagingly - a ternary relation on page 1 . We quote $[24$, p. $3 / 2]$ :

$A \rightarrow B$ is in $H_{\alpha}$ iff for every pair of set-ups $H_{\beta}$ and $H_{\gamma}$ which are $R$-related to $H_{\alpha}$ if $A$ is in $H_{\beta}$ then materially $B$ is in $H_{\gamma}$; in short, if $R\left(H_{\alpha}, H_{\beta}, H_{\gamma}\right)$ and $A$ is in $H_{\beta}$ then $B$ is in $H_{\gamma}$. Canonically relation $R$ is the following: $R\left(H_{\alpha}, H_{\beta}, H_{\gamma}\right)$ iff for every wff $B$ and $C$, if $B \rightarrow C$ is in $H_{\alpha}$ and $B$ is in $H_{\beta}$, then $C$ is in $H_{\gamma}$.

But the general implication rule requires special conditions for practically every pure implicational thesis; so while it is a fine tool for independence proofs and for systems with weak pure entailment parts, it considerably complicates first attempts to prove completeness.

According to Dunn's recollections, Meyer set out to investigate the possibility of defining a semantics for $\mathbf{R}$ through modeling $\rightarrow$ from a ternary relation in early 1971. He distinctly remembers that Meyer was working on this problem in March 1971, and we know that Urquhart received a copy of Meyer's typescript in the summer of that year. Finding a suitable modeling of implication and fusion was the missing piece in the semantical puzzle for relevance logicians at the time. We contend that Meyer's typescript contains — with a few small lapses — what later became known as the Routley-Meyer semantics for relevance logics. In particular, Meyer proves in some detail two crucial lemmas, one of which is called squeeze lemma (in later papers), together with a specialized version of it.

Meyer deals with $\mathbf{R}^{\circ \boldsymbol{t}}$ and its fragments, which is a wise choice, given the complexities that arise from $\mathbf{E}_{\rightarrow}$ 's not being formalizable by simple types of proper combinators. $\mathbf{R}^{\circ \boldsymbol{t}}$ had been algebraized by Dunn in [6] and Meyer investigated lattice- $\mathbf{R}$ in [19]. 
Meyer's manuscript was typed on a typewriter, which likely motivated certain notational choices. This may also explain why some additions were inserted as handwritten notes, and of course, there are some crossed out words and such. The typescript is way more focused and concise than Routley's manuscript - it's a mere eleven pages. We will not follow Meyer's notation and will occasionally deviate from his terminology. In order to give an idea of the main thrust of Meyer's presentation, we will give semantics for $\mathbf{R}_{\stackrel{A}{\circ}}^{\circ}, \mathbf{R}_{+}^{\circ t}$ and $\mathbf{R}^{\circ t}$.

Definition 3.1. A structure for $\mathbf{R}_{\wedge}^{\circ t}$ is a triple $\langle U, R, 0\rangle$, where $0 \in U$ and $R$ is a ternary relation on $U$ where $(\overrightarrow{\mathrm{c} 1})-(\mathrm{c} 7)$ hold. (Lower case Greek letters range over elements of $U$, and we will write $R \alpha \beta \gamma$ - as customary in the literature - instead of $R(\alpha, \beta, \gamma)$.)

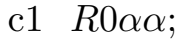

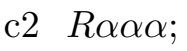

c3 $R \alpha \beta \gamma$ iff $R \beta \alpha \gamma$;

c4 $R \alpha \beta \gamma$ and $R \gamma \delta \epsilon$ imply that there is an $\eta$ such that $R \alpha \delta \eta$ and $R \eta \beta \epsilon$;

c5 $R \alpha \beta \gamma$ and $R \gamma \delta \epsilon$ imply that there is an $\eta$ such that $R \alpha \eta \epsilon$ and $R \beta \delta \eta$;

c6 $R \alpha \beta \gamma$ and $R 0 \delta \alpha$ imply that $R \delta \beta \gamma$;

c7 $R \alpha \beta \gamma$ and $R 0 \gamma \delta$ imply that $R \alpha \beta \delta .^{15}$

The set $U$ is a set of situations, which is not empty, because 0 is a distinguished element. 0 may be thought of as $\mathbf{R}_{\wedge}^{\circ \boldsymbol{t}}$ itself, which means that 0 is not an arbitrary situation. The additional conditions place restrictions on the accessibility relation, and they can be seen to correspond to certain theorems being provable in $\mathbf{R}_{\vec{A}}^{\circ \boldsymbol{t}}$.

Notation. $\alpha \leq \beta$ denotes $R 0 \alpha \beta$.

A more suggestive notation could be $R_{0} \alpha \beta$, which would stress that if we fix 0 , then the ternary relation may be viewed as a binary relation, but $\leq$ is a quite familiar relation.

\footnotetext{
${ }^{15}$ The conditions in (c5) and (c6) are our additions.
} 
Observation. The relation $\leq$ is reflexive (due to ( $\mathrm{c} 1)$ ) and transitive (due to $(\mathrm{c} 7))$. In other words, $\leq$ is a quasi-order.

The normal modal logic $\mathbf{S} \mathbf{4}$ is sound and complete for structures in which the accessibility relation is a quasi-order, which may suggest that an S4-like modality is hiding inside $\mathbf{R}_{\wedge}^{\circ t}$. The presence of a quasi-order on the set of situations suggests a potential connection to intuitionistic logic too.

Definition 3.2. A model for $\mathbf{R}_{\vec{A}}^{\circ t}$ is $\langle U, R, 0, v\rangle$, where $\langle U, R, 0\rangle$ is a structure for $\mathbf{R}_{\stackrel{\leftrightarrow}{\circ}}$, and $v$ is a valuation that maps sentence letters into increasing subsets of $U$, that is, $(\uparrow)$ holds.

$\uparrow$ If $\alpha \leq \beta$ and $\alpha \in v(p)$, then $\beta \in v(p)$.

Formulas are evaluated according to (1)-(3). (We use $\vDash$ for truth.)

$$
\begin{aligned}
& 1 \alpha, v \vDash p \quad \text { iff } \quad \alpha \in v(p) ; \\
& 2 \alpha, v \vDash \mathcal{A} \wedge \mathcal{B} \quad \text { iff } \alpha, v \vDash \mathcal{A} \text { and } \alpha, v \vDash \mathcal{B} \text {; } \\
& 3 \alpha, v \vDash \mathcal{A} \rightarrow \mathcal{B} \quad \text { iff } \quad \text { for any } \beta \text { and } \gamma, \text { if } \operatorname{Ro} \beta \gamma \text { and } \beta, v \vDash \mathcal{A} \text {, then } \\
& \gamma, v \vDash \mathcal{B} \text {. }
\end{aligned}
$$

Meyer uses the notions of truth and falsity for all formulas - with respect to a situation, and he would write $v(p, \alpha)=T$ where we have $\alpha \in v(p)$. There is a certain similarity here to the possible-world semantics that was designed by Kripke for modal logics, and later on, for intuitionistic logic.

Definition 3.3. A formula $\mathcal{A}$ is true in a structure when $0, v \vDash \mathcal{A}$ with any $v$. A formula $\mathcal{A}$ is valid when it is true in every structure for $\mathbf{R}_{\stackrel{A}{\circ}}$.

Both Dunn in [6] and Meyer in [19] allotted considerable attention to $\mathbf{R}$, and they knew that adding $\circ$ and $\boldsymbol{t}$ was conservative and a useful move. In the algebraization of $\mathbf{R}^{\circ \boldsymbol{t}}$ by Dunn, $\boldsymbol{t}$ and $\circ$ were used, and in the relational semantics Meyer used $\circ$ to define $R$. We could prove that the semantics as we have defined it so far gives soundness and completeness, however, we will follow Meyer's text more closely by listing the truth conditions for three more logical components that do not require changing the frame. In other words, the notions of a structure, a model and truth defined for $\mathbf{R}_{\vec{\wedge}}^{\circ \boldsymbol{t}}$ can be adopted for $\mathbf{R}_{+}^{\circ \boldsymbol{t}}$ without modifications.

Definition 3.4. The valuation conditions for other formulas in the language of $\mathbf{R}_{+}^{\boldsymbol{t} \circ}$ are (4)-(6).

Australasian Journal of Logic (15:2) 2018, Article no. 3.2 


$$
\begin{aligned}
& 4 \alpha, v \vDash t \quad \text { iff } \quad 0 \leq \alpha \text {; } \\
& 5 \alpha, v \vDash \mathcal{A} \circ \mathcal{B} \text { iff there are } \beta, \gamma \text { such that } R \beta \gamma \alpha \text { and } \beta, v \vDash \mathcal{A} \text { and } \\
& \gamma, v \vDash \mathcal{B} \text {; } \\
& 6 \alpha, v \vDash \mathcal{A} \vee \mathcal{B} \quad \text { iff } \quad \alpha, v \vDash \mathcal{A} \text { or } \alpha, v \vDash \mathcal{B} \text {. }
\end{aligned}
$$

Propositions are sets of possible worlds (modally speaking), and they are upward-closed sets of situations for $\mathbf{R}$. It may appear that we arbitrarily use $\leq$ that we already have in the semantics; this impression may be intensified by the lack of a similar requirement in the operational semantics discussed in Section 2. However, if $\alpha \leq \beta$ (without $\alpha=\beta$ ) is possible, then in the completeness theorem, where $\mathcal{A} \in \alpha$ iff $\alpha, v \vDash \mathcal{A}$ is proved, $\alpha, v \vDash \mathcal{A}$ should imply $\beta, v \vDash \mathcal{A}$. The following lemma became a fixture in the literature on relational semantics (cf. [8, p. 202]) - though Meyer did not prove this lemma in [20].

Lemma 3.5. For any formula of $\mathbf{R}_{+}^{\circ t}$, if $\alpha \leq \beta$ and $\alpha, v \vDash \mathcal{A}$, then $\beta, v \vDash \mathcal{A}$.

Proof. We use induction on the structure of the formulas. For sentence letters, the claim is part of the definition of a valuation. For $\boldsymbol{t}, \beta, v \vDash \boldsymbol{t}$ follows by the transitivity of $\leq$ from $\alpha, v \vDash t$ and $\alpha \leq \beta$.

Let us assume that the claim holds for $\mathcal{A}$ and $\mathcal{B}$. Then the cases of $\mathcal{A} \wedge \mathcal{B}$ and $\mathcal{A} \vee \mathcal{B}$ are straightforward (and omitted). For $\mathcal{A} \rightarrow \mathcal{B}$, let us assume that $\alpha, v \vDash \mathcal{A} \rightarrow \mathcal{B}$ and $\alpha \leq \delta$. The former implies that $\gamma, v \vDash \mathcal{B}$ is true or $R \alpha \beta \gamma$ is false or $\beta, v \vDash \mathcal{A}$ does not hold. In the first and third alternatives, obviously, $\delta, v \vDash \mathcal{A} \rightarrow \mathcal{B}$. If $R \alpha \beta \gamma$ fails, then so does $R \delta \beta \gamma$ by (c6). The case of $\mathcal{A} \circ \mathcal{B}$ uses (c7) in a similar fashion (and we omit its details).

Theorem 3.6. The logic $\mathbf{R}_{+}^{\circ t}$ is sound for the class of structures for $\mathbf{R}_{\stackrel{\wedge}{\circ}}^{\rightarrow}$.

Proof. The proof has a familiar structure: it can be shown that all axioms are valid and the rules preserve validity. We will not include all the details, because the omitted steps are either routine or can be found elsewhere. As sample steps, we consider (A1) and (A3).

1. Let us assume that $\mathcal{A} \rightarrow \mathcal{A}$ is not valid, that is, in a structure for $\mathbf{R}_{\wedge}^{\circ \boldsymbol{t}}$, with some $v, 0, v \not \models \mathcal{A} \rightarrow \mathcal{A}$. By (3) from Definition 3.2, we get that $\alpha, v \vDash \mathcal{A}$ and $R 0 \alpha \beta$, but $\beta, v \not \models \mathcal{A}$, for some $\alpha$ and $\beta$. Lemma 3.5 guarantees that $\beta, v \vDash \mathcal{A}$, which is a contradiction.

2. Again, we start with an assumption to the contrary, that is, $0, v \not \models(\mathcal{A} \rightarrow$ $(\mathcal{A} \rightarrow \mathcal{B})) \rightarrow(\mathcal{A} \rightarrow \mathcal{B})$. Then $R 0 \alpha \beta$ and $\alpha, v \vDash \mathcal{A} \rightarrow(\mathcal{A} \rightarrow \mathcal{B})$ while $\beta, v \not \models \mathcal{A} \rightarrow \mathcal{B}$. By Lemma 3.5, $\alpha, v \not \models \mathcal{A} \rightarrow \mathcal{B}$, that is, $\operatorname{R\alpha } \gamma \delta$ and $\gamma, v \vDash \mathcal{A}$ 
whereas $\delta, v \not \models \mathcal{B}$, for some $\gamma$ and $\delta$. The condition that corresponds to (A3) is $R \alpha \gamma \delta$ implies $R(\alpha \gamma) \gamma \delta .^{16}$ Meyer's list does not contain a condition of this exact shape, but here is how we can get it from $(\mathrm{c} 1)-(\mathrm{c} 7)$.

We got above that $R \alpha \gamma \delta$ and (c2) adds $R \gamma \gamma \gamma$ to this. That is, we have $R \alpha(\gamma \gamma) \delta$, hence, by (c3), $R(\gamma \gamma) \alpha \delta$. Having applied (c5) and then (c3) again, we get $R(\gamma \alpha) \gamma \delta$ and $R(\alpha \gamma) \gamma \delta$. In sum, $R \alpha \gamma \delta$ implies $R \alpha \gamma \epsilon$ and $R \epsilon \gamma \delta$, for some $\epsilon$.

From the calculations above, we know that $\alpha$ makes the antecedent of (A3) true. Then (3) from Definition 3.2 together with what we already have imply that $\epsilon, v \vDash \mathcal{A} \rightarrow \mathcal{B}$. Using $\gamma, v \vDash \mathcal{A}$ and $\operatorname{R\epsilon } \delta$, we deduce that $\delta, v \vDash \mathcal{B}$. This is a contradiction.

Definition 3.7. A set of formulas $\mathbb{T}$ is an intensional theory (ith, for short) when it is closed under (R2) and (R1) with the major premise being a provable formula. That is, (1)-(2) hold.

1 If $\mathcal{A}, \mathcal{B} \in \mathbb{T}$, then $\mathcal{A} \wedge \mathcal{B} \in \mathbb{T}$.

2 If $\mathcal{A} \in \mathbb{T}$ and $\mathcal{A} \rightarrow \mathcal{B}$ is a theorem, then $\mathcal{B} \in \mathbb{T}$.

An intensional theory is a prime intensional theory (pith, for short) when it satisfies (3) in addition to (1) and (2).

3 If $\mathcal{A} \vee \mathcal{B} \in \mathbb{T}$, then $\mathcal{A} \in \mathbb{T}$ or $\mathcal{B} \in \mathbb{T}$.

It is useful to have the following definition and lemma ready for later use. The set of formulas is an operational structure, and we can use $\circ$ on ith's by applying the connective to formulas that are elements of the ith's and by appealing to theorems (for order).

Definition 3.8. Let $\alpha$ and $\beta$ be ith's. $\alpha \circ \beta=\{\mathcal{C}: \exists \mathcal{A} \in \alpha \exists \mathcal{B} \in \beta \vdash$ $(\mathcal{A} \circ \mathcal{B}) \rightarrow \mathcal{C}\}$

Lemma 3.9. If $\alpha$ and $\beta$ are ith's, then so is $\alpha \circ \beta$. Furthermore, for any ith's $\alpha$ and $\beta$, and for any formula $\mathcal{C}, \mathcal{C} \in \alpha \circ \beta$ iff there are $\mathcal{A} \in \alpha$ and $\mathcal{B} \in \beta$ such that $\vdash(\mathcal{A} \circ \mathcal{B}) \rightarrow \mathcal{C}$.

Proof. A suitable instance of (A2) guarantees that if $\vdash \mathcal{C} \rightarrow \mathcal{D}$, then $\mathcal{C} \in \alpha \circ \beta$ implies $\mathcal{D} \in \alpha \circ \beta$. Let $\mathcal{C}_{1}$ and $\mathcal{C}_{2}$ be elements of $\alpha \circ \beta$. Then there are $\mathcal{A}_{1}, \mathcal{B}_{1}$ and $\mathcal{A}_{2}, \mathcal{B}_{2}$ such that $\vdash \mathcal{A}_{1} \circ \mathcal{B}_{1} \rightarrow \mathcal{C}_{1}$ and $\vdash \mathcal{A}_{2} \circ \mathcal{B}_{2} \rightarrow \mathcal{C}_{2}$, where the $\mathcal{A}$ 's are in $\alpha$, the $\mathcal{B}$ 's are in $\beta$. Since ith's are closed under (R2), $\mathcal{A}_{1} \wedge \mathcal{A}_{2} \in \alpha$,

\footnotetext{
${ }^{16}$ Grouping indicates the composition of $R$ with itself as usual. $R(\alpha \beta) \gamma \delta$ is shorthand for $R \epsilon \gamma \delta$ and $R \alpha \beta \epsilon$, for some $\epsilon$.
}

Australasian Journal of Logic (15:2) 2018, Article no. 3.2 
and similarly, $\mathcal{B}_{1} \wedge \mathcal{B}_{2} \in \beta$. However, $\vdash\left(\left(\mathcal{A}_{1} \wedge \mathcal{A}_{2}\right) \circ\left(\mathcal{B}_{1} \wedge \mathcal{B}_{2}\right)\right) \rightarrow \mathcal{C}_{1}$ and $\vdash\left(\left(\mathcal{A}_{1} \wedge \mathcal{A}_{2}\right) \circ\left(\mathcal{B}_{1} \wedge \mathcal{B}_{2}\right)\right) \rightarrow \mathcal{C}_{2}$, hence, $\vdash\left(\left(\mathcal{A}_{1} \wedge \mathcal{A}_{2}\right) \circ\left(\mathcal{B}_{1} \wedge \mathcal{B}_{2}\right)\right) \rightarrow\left(\mathcal{C}_{1} \wedge \mathcal{C}_{2}\right)$.

The right-to-left direction of the second claim is simply the definition of $\alpha \circ \beta$. For the left-to-right direction, we consider rules (R1) and (R2). Given what we have already said, it is sufficient to note that proofs are finite sequences of formulas, and (r1) and (r2) are theorems.

$$
\begin{aligned}
\text { r1 } \quad((\mathcal{A} \circ \mathcal{B}) \rightarrow \mathcal{C}) \rightarrow & ((\mathcal{C} \rightarrow \mathcal{D}) \rightarrow((\mathcal{A} \circ \mathcal{B}) \rightarrow \mathcal{D})) \\
\text { r2 } \quad\left(\left(\mathcal{A}_{1} \circ \mathcal{B}_{1}\right) \rightarrow \mathcal{C}_{1}\right) \rightarrow & \left(\left(\left(\mathcal{A}_{2} \circ \mathcal{B}_{2}\right) \rightarrow \mathcal{C}_{2}\right) \rightarrow\right. \\
& \left.\left(\left(\left(\mathcal{A}_{1} \wedge \mathcal{A}_{2}\right) \circ\left(\mathcal{B}_{1} \wedge \mathcal{B}_{2}\right)\right) \rightarrow\left(\mathcal{C}_{1} \wedge \mathcal{C}_{2}\right)\right)\right)
\end{aligned}
$$

For the completeness of $\mathbf{R}_{\wedge}^{\circ \boldsymbol{t}}$, ith are used, since $\boldsymbol{p} \boldsymbol{i t h}$ are undefined in

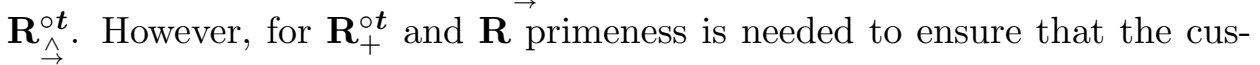
tomary clause for disjunction can be used.

Definition 3.10. Let $\mathcal{J}$ be the set of $\boldsymbol{i t h}$ in $\mathbf{R}_{\wedge}^{\circ \boldsymbol{t}}$, and let 0 be the set of theorems of $\mathbf{R}_{\wedge}^{\circ t}$. If $\alpha, \beta$ and $\gamma$ are $i t h$ 's, then $R$ holds between them in that order, that is, $\vec{R} \alpha \beta \gamma$ iff for all $\mathcal{A}$ and $\mathcal{B}$, if $\mathcal{A} \in \alpha$ and $\mathcal{B} \in \beta$, then $\mathcal{A} \circ \mathcal{B} \in \gamma$.

Lemma 3.11. The triple $\langle\mathcal{J}, 0, R\rangle$, as in Definition 3.10, is a structure for $\mathbf{R}_{\stackrel{\wedge}{\rightarrow}}^{\circ}$.

Proof. We do not include all the details here; rather, we point out that 0 is closed under (R2) and (R1), that is, $0 \in \mathcal{J}$. The bulk of the proof is to show that the accessibility relation $R$ satisfies conditions (c1)-(c7). We include details of showing that (c1), (c3) and (c6) hold.

1. Let us assume that $\mathcal{A} \in \alpha$, and $\mathcal{B}$ is an arbitrary theorem. Then, by (R3) $\boldsymbol{t} \rightarrow \mathcal{B}$ is a theorem too. Furthermore, $\boldsymbol{t} \rightarrow(\mathcal{A} \rightarrow(\mathcal{B} \circ \mathcal{A}))$ is a theorem, and by (R5) we also have that $(\boldsymbol{t} \circ \mathcal{A}) \rightarrow(\mathcal{B} \circ \mathcal{A})$ is a theorem. Also, $\mathcal{A} \rightarrow(\boldsymbol{t} \circ \mathcal{A})$ is a theorem for an arbitrary formula $\mathcal{A}$, hence, $t \circ \mathcal{A} \in \alpha$, because $\alpha$ is an ith. The transitivity of $\rightarrow$ (i.e., (A2)) gives that $\mathcal{A} \rightarrow(\mathcal{B} \circ \mathcal{A})$ is a theorem whenever $\mathcal{B}$ itself is a theorem. That is, $\mathcal{B} \circ \mathcal{A} \in \alpha$ as we intended to show. 2. For (c3), let us assume that $\mathcal{A} \in \alpha$ and $\mathcal{B} \in \beta$, hence, $\mathcal{A} \circ \mathcal{B} \in \gamma$. We have the following series of theorems $(\mathcal{B} \circ \mathcal{A}) \rightarrow(\mathcal{B} \circ \mathcal{A})$ (by $(\mathrm{A} 1))$, $\mathcal{B} \rightarrow(\mathcal{A} \rightarrow(\mathcal{B} \circ \mathcal{A}))($ by $(\mathrm{R} 6)),(\mathcal{B} \rightarrow(\mathcal{A} \rightarrow(\mathcal{B} \circ \mathcal{A}))) \rightarrow(\mathcal{A} \rightarrow(\mathcal{B} \rightarrow(\mathcal{B} \circ \mathcal{A})))$ (by $(\mathrm{A} 4)), \mathcal{A} \rightarrow(\mathcal{B} \rightarrow(\mathcal{B} \circ \mathcal{A}))$ (by $(\mathrm{R} 1))$, and finally, $(\mathcal{A} \circ \mathcal{B}) \rightarrow(\mathcal{B} \circ \mathcal{A})$ (by (R5)). Then, $\mathcal{B} \circ \mathcal{A} \in \gamma$, as needed.

3. To show that (c6) holds, we assume $R \alpha \beta \gamma$ and $R 0 \delta \alpha$. Let $\mathcal{D} \in \delta$ and $\mathcal{B} \in \beta$. We note that $\mathcal{D} \rightarrow \mathcal{D}$ is a theorem, and so is, for arbitrary $\mathcal{A}$ and $\mathcal{C}$, 
$((\mathcal{A} \rightarrow \mathcal{C}) \circ \mathcal{A}) \rightarrow \mathcal{C}$. Then $R 0 \delta \alpha$ implies that $\mathcal{D} \in \alpha$, but then $\mathcal{D} \circ \mathcal{B} \in \gamma$ using the assumption $R \alpha \beta \gamma$.

The last component in obtaining the completeness is finding a valuation. Propositions are sets of situations, in general. Concretely, we want a formula to be supported by all those ith's that contain it.

Definition 3.12. The canonical valuation is $u$ defined by (1) below and extended according to (1)-(5) from Definitions 3.2 and 3.4.

$1 \alpha, u \vDash r$ iff $r \in \alpha$, where $r$ is a propositional variable $p$ or the intensional truth $\boldsymbol{t}$.

Theorem 3.13. (Completeness for $\mathbf{R}_{\wedge}^{\circ t}$ ) For any formula $\mathcal{A}$ and for any $\alpha \in \mathcal{J}, \alpha, u \vDash \mathcal{A}$ iff $\mathcal{A} \in \alpha$.

Proof. To start with, we note that the claim is true by definition for atomic formulas. We have three connectives, hence, three cases to consider.

1. Let us assume that the claim holds for $\mathcal{A}$ and $\mathcal{B}$. $\alpha, u \vDash \mathcal{A} \wedge \mathcal{B}$ means that $\alpha, u \vDash \mathcal{A}$ and $\alpha, u \vDash \mathcal{B}$. By the hypothesis, $\mathcal{A} \in \alpha$ and $\mathcal{B} \in \alpha$, and then $\mathcal{A} \wedge \mathcal{B} \in \alpha$, because $\alpha$ is an ith. For the converse, it is sufficient to note that (A6) and (A7) are theorems. Then, $\mathcal{A} \wedge \mathcal{B} \in \alpha$ yields $\mathcal{A}, \mathcal{B} \in \alpha$, because $\alpha$ is an ith. The inductive hypothesis gives $\alpha, u \vDash \mathcal{A}$ and $\alpha, u \vDash \mathcal{B}$, that is, $\alpha, u \vDash \mathcal{A} \wedge \mathcal{B}$.

2. Our assumption for this case is the same. If $\alpha, u \not \models \mathcal{A} \rightarrow \mathcal{B}$, then for some $\beta$ and $\gamma, R \alpha \beta \gamma$ and $\beta, u \vDash \mathcal{A}$ but $\gamma, u \not \models \mathcal{B}$. By hypothesis, $\mathcal{A} \in \beta$ and $\mathcal{B} \notin \gamma$. Let $\mathcal{C} \in \alpha$, hence, $\mathcal{C} \circ \mathcal{A} \in \gamma$. If $\mathcal{A} \rightarrow \mathcal{B}$ were an element of the ith $\alpha$, then instantiating $\mathcal{C},(\mathcal{A} \rightarrow \mathcal{B}) \circ \mathcal{A}$ would be in $\gamma$. However, $((\mathcal{A} \rightarrow \mathcal{B}) \circ \mathcal{A}) \rightarrow \mathcal{B}$ is a theorem, and then $\mathcal{B}$ must have been in $\gamma$ leading to a contradiction. Therefore, $\mathcal{A} \rightarrow \mathcal{B} \notin \alpha$. To prove the other half, let us suppose that $\mathcal{A} \rightarrow \mathcal{B} \notin \alpha$. We will consider two sets of formulas in place of the $\boldsymbol{i t h}$ 's we need. Let $\beta$ be the $\boldsymbol{i t h}$ generated by $\mathcal{A}$. To find a suitable $\gamma$, we consider $\alpha \circ \beta$. Clearly, $R \alpha \beta \gamma$. To see that $\mathcal{B} \in \gamma$ is impossible, let us assume that it is the case. Then, there is a $\mathcal{C} \in \alpha$ such that $\vdash(\mathcal{C} \circ \mathcal{A}) \rightarrow \mathcal{B}$, which means that $\vdash \mathcal{C} \rightarrow(\mathcal{A} \rightarrow \mathcal{B})$. However, $\alpha$ is an ith, and $\mathcal{A} \rightarrow \mathcal{B} \notin \alpha$. Since $\mathcal{B} \notin \gamma$, we get that $\alpha, u \not \models \mathcal{A} \rightarrow \mathcal{B}$.

3. Once again, we assume that the claim holds for the immediate proper subformulas of $\mathcal{A} \circ \mathcal{B}$. Let $\alpha, u \vDash \mathcal{A} \circ \mathcal{B}$, that is, $\beta, u \vDash \mathcal{A}$ and $\gamma, u \vDash \mathcal{B}$ for some $\beta$, $\gamma$ such that $R \beta \gamma \alpha$. Since $\mathcal{A} \in \beta$ and $\mathcal{B} \in \gamma$, the definition of $R$ gives that $\mathcal{A} \circ \mathcal{B} \in \alpha$. For the other direction, we start by assuming the latter. We must find a pair of $\boldsymbol{i t h}$ 's that can be taken in the places of $\beta$ and $\gamma$. Let $\beta$ be the $\boldsymbol{i t h}$ generated by $\mathcal{A}$, that is, $\beta=\{\mathcal{C}: \vdash \mathcal{A} \rightarrow \mathcal{C}\}$. Similarly, let $\gamma$ 
be generated by $\mathcal{B}$. We note that (A18) guarantees closure under rule (R2). The tonicity property of $\circ$ with respect to provable $\rightarrow$ makes sure that for all $\mathcal{C} \in \beta$ and for all $\mathcal{D} \in \gamma, \mathcal{C} \circ \mathcal{D} \in \alpha$. This means that $R \beta \gamma \alpha$ holds, hence, $\alpha, u \vDash \mathcal{A} \circ \mathcal{B}$, as we wanted to show.

Meyer considered the completeness for $\mathbf{R}_{A}^{\circ t}$ separately, because the claim we just proved cannot be proved for formulas of the form $\mathcal{A} \vee \mathcal{B}$. The reason is that any formula generates its own $\boldsymbol{i t h}$, which however, will not include any formulas that are distinct from the given formula while they imply the given formula. If $\mathcal{A}$ and $\mathcal{B}$ are distinct, and $(\mathcal{A} \vee \mathcal{B}) \rightarrow \mathcal{A}$ and $(\mathcal{A} \vee \mathcal{B}) \rightarrow \mathcal{B}$ are not theorems, then the $i$ th generated by $\mathcal{A} \vee \mathcal{B}$ contains the formula itself, but does not contain either $\mathcal{A}$ or $\mathcal{B}$. This is the reason to change Definition 3.10.

Definition 3.14. Let $\mathcal{J}^{\prime}$ be the set of pith in $\mathbf{R}_{+}^{\circ \boldsymbol{t}}$, and let 0 be the set of theorems of $\mathbf{R}_{+}^{\circ \boldsymbol{t}}$. If $\alpha, \beta$ and $\gamma$ are pith's, then $R$ holds between them in that order, that is, $R \alpha \beta \gamma$ iff for all $\mathcal{A}$ and $\mathcal{B}$, if $\mathcal{A} \in \alpha$ and $\mathcal{B} \in \beta$, then $\mathcal{A} \circ \mathcal{B} \in \gamma$.

Now we have to prove anew that $\left\langle\mathcal{J}^{\prime}, R, 0\right\rangle$ is a structure for $\mathbf{R}_{\wedge}^{\circ t}$ (hence, for $\mathbf{R}_{+}^{\circ \boldsymbol{t}}$ too) in the sense of Definition 3.1. The conditions that appear to be the most difficult to prove are (c4) and (c5), because in each, a suitable pith has to be found. Meyer's strategy is to prove two lemmas, which are useful in several places in the completeness proof. Indeed, they capture significant insights about the algebras of $\mathbf{R}_{\stackrel{\wedge}{\circ}}$ and $\mathbf{R}_{+}^{\circ t}$.

Lemma 3.15. If $\alpha$ is an ith, and $\mathcal{B}$ is a formula, then let $\alpha \mathrm{uB}$ be the least ith such that $\alpha \cup\{\mathcal{B}\} \subseteq \alpha \mathrm{uB}$. For any $\mathcal{C} \in \alpha u \mathcal{B}$, there is an $\mathcal{A} \in \alpha$ such that $\vdash(\mathcal{A} \wedge \mathcal{B}) \rightarrow \mathcal{C}$.

Proof. The cases when $\mathcal{C} \in \alpha$, or $\mathcal{C}$ is $\mathcal{B}$ are obvious. We have to make sure that the claim is inherited through finitely many applications of the rules that constitute the closure conditions on ith's.

1. Let $\vdash \mathcal{C}_{1} \rightarrow \mathcal{C}_{2}$ whereas $\mathcal{C}_{1} \in \alpha$ uB . By hypothesis, $\vdash(\mathcal{A} \wedge \mathcal{B}) \rightarrow \mathcal{C}_{1}$, and by an instance of (A2) and the definition of ith, $\vdash(\mathcal{A} \wedge \mathcal{B}) \rightarrow \mathcal{C}_{2}$.

2. If $\mathcal{C}_{1}, \mathcal{C}_{2} \in \alpha \mathrm{u} \mathcal{B}$, then $\vdash\left(\mathcal{A}_{1} \wedge \mathcal{B}\right) \rightarrow \mathcal{C}_{1}$ as well as $\vdash\left(\mathcal{A}_{2} \wedge \mathcal{B}\right) \rightarrow \mathcal{C}_{2}$. Using axioms (A6)-(A8) together with the transitivity of $\rightarrow$, that is, (A2), we obtain that $\vdash\left(\mathcal{A}_{1} \wedge \mathcal{A}_{2} \wedge \mathcal{B}\right) \rightarrow \mathcal{C}_{1}$ and $\vdash\left(\mathcal{A}_{1} \wedge \mathcal{A}_{2} \wedge \mathcal{B}\right) \rightarrow \mathcal{C}_{2}$. Then, (A8) gives $\vdash\left(\mathcal{A}_{1} \wedge \mathcal{A}_{2} \wedge \mathcal{B}\right) \rightarrow\left(\mathcal{C}_{1} \wedge \mathcal{C}_{2}\right)$. Of course, we assumed that the $\mathcal{A}$ 's were in $\alpha$, hence, $\mathcal{A}_{1} \wedge \mathcal{A}_{2} \in \alpha$ too. 
Lemma 3.16. (Squeeze lemma) If $\alpha \circ \beta \subseteq \gamma$, where $\alpha, \beta \in \mathcal{J}$ and $\gamma \in \mathcal{J}^{\prime}$, then there is an $\alpha^{\prime} \in \mathcal{J}^{\prime}$ such that $\alpha \subseteq \alpha^{\prime}$ and $\alpha^{\prime} \circ \beta \subseteq \gamma$.

Proof. First of all, note that even if o were not commutative, all the steps in the proof would show the truth of the claim with $\alpha$ and $\beta$ interchanged. Of course, for $\mathbf{R}$ this observation is unimportant, but it opens up the possibility of adapting the semantics to other logics, notably, to $\mathbf{E}$.

Let $\mathbb{A}=\{\delta \in \mathcal{J}: \alpha \subseteq \delta \wedge \delta \circ \beta \subseteq \gamma\} .{ }^{17} \mathbb{A} \neq \emptyset$, because $\alpha \in \mathbb{A}$. The union of a chain (with respect to $\subseteq$ ) from $\mathbb{A}$ is an element of $\mathbb{A}$ and an upper bound for the chain. Hence, by Zorn's lemma, there is a maximal element in $\mathbb{A}$.

The point of getting a maximal (within $\mathbb{A}$ ) ith is that it can be shown to be prime. Let's assume that for some $\mathcal{A}_{1}$ and $\mathcal{A}_{2},\left(\alpha \mathrm{u} \mathcal{A}_{1}\right) \circ \beta \nsubseteq \gamma$, and $\left(\alpha \mathrm{u} \mathcal{A}_{2}\right) \circ \beta \nsubseteq \gamma$. By Lemma 3.9, there are $\mathcal{C}_{1} \in \alpha \mathrm{u} \mathcal{A}_{1}, \mathcal{C}_{2} \in \alpha \mathrm{u} \mathcal{A}_{2}$ and $\mathcal{B}_{1}, \mathcal{B}_{2} \in \beta$ such that $\mathcal{C}_{1} \circ \mathcal{B}_{1} \notin \gamma$ and $\mathcal{C}_{2} \circ \mathcal{B}_{2} \notin \gamma$. Let $\mathcal{B}$ be the conjunction of the $\mathcal{B}$ 's; then, $\mathcal{C}_{1} \circ \mathcal{B} \notin \gamma, \mathcal{C}_{2} \circ \mathcal{B} \notin \gamma$. By Lemma 3.15, we know that there are $\mathcal{D}_{1}$ and $\mathcal{D}_{2}$ such that $\vdash \mathcal{A}_{1} \wedge \mathcal{D}_{1} \rightarrow \mathcal{C}_{1}$ and $\vdash \mathcal{A}_{2} \wedge \mathcal{D}_{2} \rightarrow \mathcal{C}_{2}$. Let $\mathcal{D}$ be the conjunction of the $\mathcal{D}$ 's. Combining all the formulas we have, we get that $\left(\mathcal{A}_{1} \wedge \mathcal{D}\right) \circ \mathcal{B} \notin \gamma$, neither is $\left(\mathcal{A}_{2} \wedge \mathcal{D}\right) \circ \mathcal{B} \in \gamma$. $\gamma$ is a pith, thus, $\left(\left(\mathcal{A}_{1} \wedge \mathcal{D}\right) \circ \mathcal{B}\right) \vee\left(\left(\mathcal{A}_{2} \wedge \mathcal{D}\right) \circ \mathcal{B}\right) \notin \gamma$ either. However, there are pleasing interactions between the connectives in $\mathbf{R}_{+}^{\circ \text { t }}$; in particular, $\vdash\left(\left(\left(\mathcal{A}_{1} \wedge \mathcal{D}\right) \vee\right.\right.$ $\left.\left.\left(\mathcal{A}_{2} \wedge \mathcal{D}\right)\right) \circ \mathcal{B}\right) \rightarrow\left(\left(\left(\mathcal{A}_{1} \wedge \mathcal{D}\right) \circ \mathcal{B}\right) \vee\left(\left(\mathcal{A}_{2} \wedge \mathcal{D}\right) \circ \mathcal{B}\right)\right)$ and $\vdash\left(\left(\left(\mathcal{A}_{1} \vee \mathcal{A}_{2}\right) \wedge \mathcal{D}\right) \circ \mathcal{B}\right) \rightarrow$ $\left(\left(\left(\mathcal{A}_{1} \wedge \mathcal{D}\right) \vee\left(\mathcal{A}_{2} \wedge \mathcal{D}\right)\right) \circ \mathcal{B}\right)$. Since $\left(\left(\left(\mathcal{A}_{1} \vee \mathcal{A}_{2}\right) \wedge \mathcal{D}\right) \circ \mathcal{B}\right) \notin \gamma$, we can conclude that $\mathcal{A}_{1} \vee \mathcal{A}_{2} \notin \delta$.

The squeeze lemma is useful not only in the proof of the next lemma, but also in the proof of the completeness theorem.

Lemma 3.17. The triple $\left\langle\mathcal{J}^{\prime}, R, 0\right\rangle$ is a structure for $\mathbf{R}_{+}^{\circ t}$.

Proof. The set of theorems of $\mathbf{R}_{+}^{\circ \boldsymbol{t}}$ is a pith. An easy way to prove this (and we omit the details) is by using the sequent calculus $L \mathbf{R}_{+}$from Dunn [7]. ${ }^{18}$ The bulk of the proof is to show that $R$ defined as before but restricted to $\mathrm{J}^{\prime}$ has the properties captured by $(\mathrm{c} 1)-(\mathrm{c} 7)$. Informally speaking, the crux is whether there are sufficiently many pith's of the desired shape. That is, we are interested not merely in the cardinal number for $\mathcal{J}^{\prime}$, but we want to have pith's to be nicely dispersed. We consider (c2) and (c4) in some detail, and leave the other conditions for the reader to check.

\footnotetext{
${ }^{17}$ Meyer considers a larger set of $i t h$ 's in the proof by omitting the inclusion of $\alpha$ into $\delta$.

${ }^{18}$ Dunn presented $L \mathbf{R}_{+}$at a conference in 1969 . This sequent calculus is one of the justifications Meyer gives for 0 being a pith - together with a result of his own.
}

Australasian Journal of Logic (15:2) 2018, Article no. 3.2 
1. Let us assume that $\mathcal{A}, \mathcal{B} \in \alpha$. Then $\mathcal{B} \rightarrow(\mathcal{A} \circ \mathcal{B}) \in \alpha$, because $\alpha \in \mathcal{J}^{\prime}$ and $\vdash \mathcal{A} \rightarrow(\mathcal{B} \rightarrow(\mathcal{A} \circ \mathcal{B}))$. Also, $\mathcal{B} \wedge(\mathcal{B} \rightarrow(\mathcal{A} \circ \mathcal{B})) \in \alpha$. Another theorem of $\mathbf{R}_{+}^{\circ t},(\mathcal{B} \wedge(\mathcal{B} \rightarrow(\mathcal{A} \circ \mathcal{B}))) \rightarrow(\mathcal{A} \circ \mathcal{B})$ guarantees that $\mathcal{A} \circ \mathcal{B} \in \alpha$, as desired. 2. For (c4), let us assume both $R \alpha \beta \gamma$ and $R \gamma \delta \epsilon$. Let $\mathcal{A} \in \alpha, \mathcal{B} \in \beta$ and $\mathcal{D} \in \delta . R(\alpha \circ \delta) \beta \epsilon$, because - using our arbitrary formulas - $(\mathcal{A} \circ \mathcal{B}) \circ \mathcal{D} \in \epsilon$, but $\vdash((\mathcal{A} \circ \mathcal{B}) \circ \mathcal{D}) \rightarrow((\mathcal{A} \circ \mathcal{D}) \circ \mathcal{B})$. By Lemma 3.9, $\alpha \circ \delta$ is an ith, and by the Squeeze Lemma, there is a pith $\eta$ such that $R \eta \beta \epsilon$ and $\alpha \circ \delta \subseteq \eta$. Such an $\eta$, by its construction, possesses the property that $R \alpha \delta \eta$ holds of it.

Before we turn to proving completeness, it is useful to single out another lemma, which captures a deep insight into what sorts of $\boldsymbol{i t h}$ are pith.

Lemma 3.18. If $\alpha$ is an ith with the property that $\mathcal{A} \notin \alpha$, then there is a pith $\gamma$ that extends $\alpha$ without including $\mathcal{A}$.

Proof. We maximize $\alpha$, and then we show that the resulting ith is a pith. Let $E$ be $\{\beta: \alpha \subseteq \beta \wedge \beta \in \mathcal{J} \wedge \mathcal{A} \notin \beta\}$. Obviously, $\alpha \in E$, and the union of non-empty chains is an upper bound in $E$. Let $\gamma$ be a maximal element in $E$, by Zorn's lemma. To show primeness, we argue that if $\mathcal{B} \notin \gamma$ and $\mathcal{C} \notin \gamma$, then neither is $\mathcal{B} \vee \mathcal{C} \in \gamma$. There are $\mathcal{D}_{1}$ and $\mathcal{D}_{2}$ in $\alpha$ such that $\vdash\left(\mathcal{D}_{1} \wedge \mathcal{B}\right) \rightarrow \mathcal{A}$ and $\vdash\left(\mathcal{D}_{2} \wedge \mathcal{C}\right) \rightarrow \mathcal{A}$. We denote $\mathcal{D}_{1} \wedge \mathcal{D}_{2}$ by $\mathcal{D} ; \mathcal{D} \in \alpha$, because $\alpha$ is an ith. Then, $(\mathcal{D} \wedge \mathcal{B}) \rightarrow\left(\mathcal{D}_{1} \wedge \mathcal{B}\right)$ and similarly, for $\mathcal{D}_{2}$ and $\mathcal{C}$. By suffixing and detachment, $\vdash(\mathcal{D} \wedge \mathcal{B}) \rightarrow \mathcal{A}$ and $\vdash(\mathcal{D} \wedge \mathcal{C}) \rightarrow \mathcal{A}$. Using (R2), the distribution of $\rightarrow$ and $\wedge$ over $\vee$, we get that $\vdash(\mathcal{D} \wedge(\mathcal{C} \vee \mathcal{B})) \rightarrow \mathcal{A}$. This implies that $\mathcal{B} \vee \mathcal{C} \notin \gamma$ either.

Theorem 3.19. (Completeness for $\mathbf{R}_{+}^{\circ t}$ ) If $0, v \vDash \mathcal{A}$ in any structure for $\mathbf{R}_{+}^{\circ \boldsymbol{t}}$ under any valuation $v$, then $\mathcal{A}$ is a theorem of $\mathbf{R}_{+}^{\circ \boldsymbol{t}}$.

Proof. We use the triple $\left\langle\mathcal{J}^{\prime}, R, 0\right\rangle$ together with the earlier definition of the canonical valuation (where $\alpha$ is a variable running over $\mathcal{J}^{\prime}$ now). The cases for atomic formulas are as before; the cases for $\wedge$ and $\vee$ are easy. We consider in some detail the cases for the two other connectives.

1. Let us consider $\mathcal{A} \rightarrow \mathcal{B}$. The first half of step 2 in the proof of Theorem 3.13 stands as before. The second half requires some addition, namely, $\beta$ and $\gamma$ as defined there do not need to be pith's. However, we have established that $R \alpha \beta \gamma$ holds, while $\mathcal{B} \notin \gamma$. We appeal to Lemma 3.18, that is, to maximizing $\gamma$ on the condition that $\mathcal{B} \notin \gamma$, which yields a pith, let's say, $\gamma^{\prime}$. $\gamma \subseteq \gamma^{\prime}$ ensures that $R \alpha \beta \gamma^{\prime}$ holds too. Then, by the Squeeze Lemma, there is a pith $\beta^{\prime}$ such that $R \alpha \beta^{\prime} \gamma^{\prime}$. 
2. The situation is similar, though slightly simpler, for $\mathcal{A} \circ \mathcal{B}$. The first half of step 3 in the same proof requires two applications of the Squeeze Lemma to obtain the pith's $\beta^{\prime}$ and $\gamma^{\prime}$ from $\beta$ and $\gamma$, respectively, with $R \beta^{\prime} \gamma^{\prime} \alpha$.

This completes the proof of the completeness theorem for $\mathbf{R}_{+}^{\circ t}$.

Section X of Meyer's typescript takes care of negation. He opts for an operational modeling of $\sim$. Dunn in his [6] gave a series of set-theoretic representations for De Morgan lattices - the algebraic equivalents of fde, first-degree entailments that are a common fragment of some of the most notable relevance $\operatorname{logics} \mathbf{B}, \mathbf{T}, \mathbf{E}$ and $\mathbf{R}$ (but not of linear logic $\mathbf{L L}$ ). The operational representation, originally due to Białynicki-Birula and Rasiowa, was certainly known to Meyer, who in fact, used * for the operation. For fde, little needs to be postulated about *, namely, that it's an involution. However, in $\mathbf{R}$, there is an interplay between $\rightarrow$ and $\sim$, because of one or another form of contraposition. In the axiomatization we gave in Section 1, it is expressed by (A15). Meyer included a condition - the one that is most frequently recited today — that suffices for soundness.

Definition 3.20. A structure for $\mathbf{R}^{\circ t}$ is $\left\langle U, R, Z,{ }^{*}\right\rangle$, where (c2)-(c7) from Definition 3.1 hold together with $\left(\mathrm{c1}^{\prime}\right)$ and $(\mathrm{c} 8)-(\mathrm{c} 9)$.

$\mathrm{c}^{\prime}$ For all $\alpha$, there is a $z \in Z$ for which $R z \alpha \alpha$ holds;

$$
\begin{aligned}
& \text { c8 } \alpha^{* *}=\alpha ; \\
& \text { c9 } R \alpha \beta \gamma \text { iff } R \alpha \gamma^{*} \beta^{*} .
\end{aligned}
$$

Meyer omitted 0 or $Z$ altogether from his definition by including instead the choice of a suitable 0 into the refutation of a non-theorem in the proof of the completeness theorem. He went on to discuss how the admissibility of the rule $\gamma$ proved in [21] guaranteed the existence of a suitable 0.

Remark 3.21. We list here what is affected by $Z$ taking the place of 0 .

The notation $\leq$ is defined by existentially quantifying over elements of $Z$, that is, $\alpha \leq \beta$ iff $\exists z \in Z R z \alpha \beta$.

The satisfiability clause (4) from Definition 3.4 is similarly weakened.

$$
4^{\prime} \quad \alpha, v \vDash t \quad \text { iff } \quad \exists z \in Z z \leq \alpha .
$$

Lemma 3.5 holds for all formulas of $\mathbf{R}^{\circ}$. We quickly show that it's true for $\sim \mathcal{A}$. If $\alpha \vDash \sim \mathcal{A}$ and $\alpha \leq \beta$, then $\alpha^{*} \not \models \mathcal{A}$ (by (7) below). Also, by (c9), $\beta^{*} \leq \alpha^{*}$ and using the inductive hypothesis, $\beta^{*} \not \models \mathcal{A}$, which is to say that $\beta \vDash \sim \mathcal{A}$, as desired. 
For $\mathbf{R}_{\wedge}^{\circ t}$ or $\mathbf{R}_{+}^{\circ t}$, we could adopt $Z$ easily, because in those logics $Z=$ $\{z: 0 \leq z\}$, moreover, $0 \in Z$.

Definition 3.22. A model for $\mathbf{R}^{\circ t}$ is $\left\langle U, R, Z,{ }^{*}, v\right\rangle$, with ( $\uparrow$ ) true (from Definition 3.2), and with formulas evaluated according to (1)-(6) from Definitions 3.2 and 3.4 , and (7) below.

$$
7 \alpha, v \vDash \sim \mathcal{A} \quad \text { iff } \quad \alpha^{*}, v \not \models \mathcal{A} .
$$

Theorem 3.23. If $\mathcal{A}$ is a theorem of $\mathbf{R}^{\circ \boldsymbol{t}}$, then in any model for $\mathbf{R}^{\circ \boldsymbol{t}}$, under any valuation $v, z, v \vDash \mathcal{A}$, when $z \in Z$.

Proof. The majority of the cases is as before. We give a sample case in some detail. Let $z \notin(\mathcal{A} \rightarrow \sim \mathcal{A}) \rightarrow \sim \mathcal{A}$, that is, $\alpha \vDash \mathcal{A} \rightarrow \sim \mathcal{A}, \beta \not \models \sim \mathcal{A}$ while $R z \alpha \beta$ for some $\alpha$ and $\beta$. Since $\alpha \leq \beta$, we may use $\alpha \not \models \sim \mathcal{A}$, which yields $\alpha^{*} \vDash \mathcal{A}$. By (c2), we have that $R \alpha^{*} \alpha^{*} \alpha^{*}$, hence, by (c8)-(c9), $R \alpha^{*} \alpha \alpha$. Further, a suitable instance of (c3) gives $R \alpha \alpha^{*} \alpha$. Then $\alpha \vDash \mathcal{A} \rightarrow \sim \mathcal{A}$ means that $\alpha \vDash \sim \mathcal{A}$, that is, $\alpha^{*} \not \models \mathcal{A}$. From the contradiction, we infer that axiom (A14) is valid.

For the completeness, Meyer defined a triple $\left\langle\mathrm{J}^{\prime}, R,{ }^{*}\right\rangle$ and showed that for any non-theorem a suitable 0 (in his notation) may be found. We instead consider the union of all clusters of non-theorems (to use his terminology), that is, a $Z$ (using the notation from [12]).

Definition 3.24. Let $\mathcal{J}^{\prime}$ be the set of pith in $\mathbf{R}^{\circ t}$, and let $Z$ be the set of pith's that contain all the theorems of $\mathbf{R}^{\circ t}$. The definition of $R$ is as in Definition 3.14. If $\alpha \in \mathcal{J}^{\prime}$, then $\alpha^{*}=\{\mathcal{A}: \sim \mathcal{A} \notin \alpha\}$.

Lemma 3.25. The four-tuple $\left\langle\mathcal{J}^{\prime}, R, Z,{ }^{*}\right\rangle$ is a structure for $\mathbf{R}^{\circ t}$.

Proof. This proof can incorporate what has already been established for $\mathbf{R}_{\wedge}^{\circ t}$. Additionally, it has to be established that the modified (c1') holds together with the new conditions (c8)-(c9). It also has to be proved that * produces a pith from a pith. We omit all the details here.

The last section in [20] is devoted to showing that there is a suitable valuation on the structure in the four-tuple.

Theorem 3.26. (Completeness for $\mathbf{R}^{\circ t}$ ) If $\mathcal{A}$ is valid in all structures for $\mathbf{R}^{\circ \boldsymbol{t}}$, then $\mathcal{A}$ is a theorem of $\mathbf{R}^{\circ \boldsymbol{t}}$. 
Proof. The valuation that we want to use is the one in Definition 3.12. We will limit ourselves to showing that $\alpha, u \vDash \sim \mathcal{A}$ iff $\sim \mathcal{A} \in \alpha$. The other cases are similar to cases for $\mathbf{R}_{+}^{\circ \boldsymbol{t}}$. We give a series of iff's, with the middle iff justified by the inductive hypothesis; the others come from definitions.

$$
\sim \mathcal{A} \in \alpha \quad \text { iff } \quad \mathcal{A} \notin \alpha^{*} \quad \text { iff } \quad \alpha^{*}, u \not \models \mathcal{A} \quad \text { iff } \quad \alpha, u \vDash \sim \mathcal{A}
$$

We started this section with a quote from Routley's manuscript. It seems that Routley was correct in his assessment that the ternary relational approach would require conditions characterizing the relation itself to obtain a semantics for the various relevance logics. Of course, we may point out that the operational approach contains similar complications in the form of new entities and various principles. It seems to us that Meyer's typescript is much closer to the "Routley-Meyer semantics" than Routley's manuscript is. Although Meyer missed some conditions, he recognized the importance of pith's (possibly, having been influenced by algebraic studies of relevance logics), and he formulated and proved crucial lemmas. His work, at least in spirit, is a continuation of the Jónsson-Tarski approach to the representation of Boolean algebras with operators, and Kripke's semantics for normal modal and intuitionistic logics. In retrospect, generalized Galois logics (i.e., gaggle theory) might provide the rationale why the ternary relational approach proved to be more successful than the various semi-lattice-based semantics. ${ }^{19}$

\section{Conclusions}

Routley's manuscript is clearly pioneering; it has a broad scope and is rich with ideas. Meyer starts his typescript with acknowledgments, which likely refer to Urquhart's draft of his operational semantics and to Routley's manuscript. (The typescript does not include a list of references or pointers to concrete writings.) He claims that Dunn and he have shown that the operational semantics (with the usual clause, like (6) above) cannot be extended to a semantics for $\mathbf{R}$ (or even $\mathbf{R}_{+}$). The full appreciation of this fact and its consequences for attempts to give a semantics for $\mathbf{R}$ must have been the decisive motive to design a semantics using a ternary relation.

\section{Acknowledgments}

We would like to express our thanks to Alasdair Urquhart for providing us with a scan of Richard Routley's manuscript parts of which we discuss in

\footnotetext{
${ }^{19}$ For gaggle theory, see Dunn's papers $[9,10,11]$, and also [13, Ch. 12] and [3].
} 
this paper. We are also grateful to Michael A. McRobbie for providing us with a scan of Robert K. Meyer's typescript that we examined in Section 3. Also, we would like to thank the referees for their comments.

\section{References}

[1] Alan R. Anderson, Nuel D. Belnap, and J. Michael Dunn. Entailment: The Logic of Relevance and Necessity, volume II. Princeton University Press, Princeton, NJ, 1992.

[2] Katalin Bimbó. Relevance logics. In D. Jacquette, editor, Philosophy of Logic, volume 5 of Handbook of the Philosophy of Science (D. Gabbay, P. Thagard and J. Woods, eds.), pages 723-789. Elsevier (North-Holland), Amsterdam, 2007.

[3] Katalin Bimbó and J. Michael Dunn. Generalized Galois Logics: Relational Semantics of Nonclassical Logical Calculi, volume 188 of CSLI Lecture Notes. CSLI Publications, Stanford, CA, 2008.

[4] Katalin Bimbó and J. Michael Dunn. The emergence of set-theoretical semantics for relevance logics around 1970. In K. Bimbó and J. M. Dunn, editors, Proceedings of the Third Workshop, May 16-17, 2016, Edmonton, Canada, volume 4(3) of IFCoLog Journal of Logics and Their Applications, pages 557-589, London, UK, 2017. College Publications.

[5] B. Jack Copeland. The genesis of possible worlds semantics. Journal of Philosophical Logic, 31:99-137, 2002.

[6] J. Michael Dunn. The Algebra of Intensional Logics. PhD thesis, University of Pittsburgh, Ann Arbor (UMI), 1966.

[7] J. Michael Dunn. A 'Gentzen system' for positive relevant implication, (abstract). Journal of Symbolic Logic, 38:356-357, 1973.

[8] J. Michael Dunn. Relevance logic and entailment. In D. Gabbay and F. Guenthner, editors, Handbook of Philosophical Logic, volume 3, pages 117-224. D. Reidel, Dordrecht, 1st edition, 1986.

[9] J. Michael Dunn. Gaggle theory: An abstraction of Galois connections and residuation, with applications to negation, implication, and various 
logical operators. In J. van Eijck, editor, Logics in AI: European Workshop JELIA '90, number 478 in Lecture Notes in Computer Science, pages 31-51. Springer, Berlin, 1991.

[10] J. Michael Dunn. Partial gaggles applied to logics with restricted structural rules. In K. Došen and P. Schroeder-Heister, editors, Substructural Logics, pages 63-108. Clarendon, Oxford, UK, 1993.

[11] J. Michael Dunn. Gaggle theory applied to intuitionistic, modal and relevance logics. In I. Max and W. Stelzner, editors, Logik und Mathematik. Frege-Kolloquium Jena 1993, pages 335-368. W. de Gruyter, Berlin, 1995.

[12] J. Michael Dunn. A representation of relation algebras using RoutleyMeyer frames. In C. A. Anderson and M. Zelëny, editors, Logic, Meaning and Computation. Essays in Memory of Alonzo Church, pages 77108. Kluwer, Dordrecht, 2001.

[13] J. Michael Dunn and Gary M. Hardegree. Algebraic Methods in Philosophical Logic, volume 41 of Oxford Logic Guides. Oxford University Press, Oxford, UK, 2001.

[14] Kit Fine. Models for entailment. Journal of Philosophical Logic, 3: 347-372, 1974.

[15] Kit Fine. Incompleteness for quantified relevance logics. In J. Norman and R. Sylvan, editors, Directions in Relevant Logic, volume 1 of Reason and Argument, pages 205-225. Kluwer Academic Publishers, Dordrecht, 1989.

[16] Robert Goldblatt. Mathematical modal logic: A view of its evolution. In Dov M. Gabbay and John Woods, editors, Modalities in the Twentieth Century, volume 7 of Handbook of the History of Logic, pages 1-98. Elsevier, Amsterdam, 2006.

[17] Saul A. Kripke. Semantical considerations on modal logic. Acta Philosophica Fennica, 16:83-94, 1963. Proceedings of a colloquium on modal and many-valued logics, Helsinki, 23-26 August 1962.

[18] Larisa L. Maksimova. Struktury s implikatsiěr. Algebra i logika, 12(4): 445-467, 1973. [Structures with implication].

[19] Robert K. Meyer. Topics in Modal and Many-valued Logic. PhD thesis, University of Pittsburgh, Ann Arbor (UMI), 1966.

Australasian Journal of Logic (15:2) 2018, Article no. 3.2 
[20] Robert K. Meyer. From the desk of Robert K. Meyer: Semantical completeness of relevant implication. (11 pages, typescript), 1971.

[21] Robert K. Meyer and J. Michael Dunn. E, R and $\gamma$. Journal of Symbolic Logic, 34:460-474, 1969. Reprinted in Anderson, A. R. and N. D. Belnap, Entailment: The Logic of Relevance and Necessity, vol. 1, Princeton University Press, Princeton, NJ, 1975, §25.2, pp. 300-314.

[22] Grigoriǐ E. Mints. Cut-elimination theorem for relevant logics [in Russian]. Zapiski Nauchnykh Seminarov Leningradskogo Otdeleniya Matematicheskogo Instituta imeni V. A. Steklova AN USSR, 32:90-97, 1972. (English translation in Journal of Soviet Mathematics, 6:422-428, 1976).

[23] Greg Restall. Four-valued semantics for relevant logics (and some of their rivals). Journal of Philosophical Logic, 24:139-160, 1995.

[24] Richard Routley. Semantical analysis of relevant implication: I. (96 pages, manuscript), ca. 1970.

[25] Richard Routley. Semantical analysis of relevant implication: I. (ca. 1970), transcript by N. J. Ferenz, (70 pages), 2017.

[26] Richard Routley and Valerie Routley. The semantics of first degree entailment. Noûs, 6:335-359, 1972.

[27] Alasdair Urquhart. Semantics for relevant logic. Journal of Symbolic Logic, 37:159-169, 1972.

[28] Alasdair Urquhart. The undecidability of entailment and relevant implication. Journal of Symbolic Logic, 49:1059-1073, 1984.

[29] Richard Zach. Completeness before Post: Bernays, Hilbert, and the development of propositional logic. Bulletin of Symbolic Logic, 5:331366, 1999 . 